\title{
Prevenir en otra onda
}

\section{To prevent in another wave}

José Guillermo FOUCE 1 Mónica M. FOSSOUL PALICIO²

\section{RESUMEN}

El articulo que ahora presentamos es el resultado de la confluencia de dos grandes lineas de actuación que venimos desarrollando desde hace algunos años, por una parte, la investigación para constatar la relación entre música y drogas en las letras de un gran número de grupos musicales $y$, por otra parte, y más relevante en esta oportunidad, aprovechar esta constatación y estos diversos materiales obtenidos para desarrollar un programa preventivo novedoso que hemos denominado "Prevenir en otra onda" para jóvenes de 3 y $4^{\circ}$ de la E.S.O. y de $1^{\circ}$ y $2^{\circ}$ de Bachillerato. En el texto se presentan los resultados obtenidos en la primera ejecución de este programa preventivo, así como las principales conclusiones obtenidas.

\section{PALABRAS CLAVE}

Prevención, Drogas, Jóvenes, Música.

\section{ABSTRACT}

The article that we present here is the result of the confluence of two big lines of performance that we have being developing for years. On one hand, the research to verify the relation between music and drugs in the lyrics of a large number of musical groups and, on the other hand, and more relevant opportunity, to make use of this verification and

\footnotetext{
${ }^{1}$ Doctor en psicologia. Coordinador del CAID de Torrejón de Ardoz (Madrid, desde el 2001 a junio 2007) y presidente de Psicólogos Sin Fronteras Madrid. psf@psicologossinfronteras.net

2 Psicóloga educativa. Vicepresidenta de Psicólogos Sin Fronteras Madrid.
} 
Prevenir en otra onda

these diverse materials obtained in order to develop a new preventive program named "To prevent in another wave "for young men of 3rd and 4th of the ESO and with 1st and 2nd of BUP. In the text we present the results of first performance of this preventive program, as well as the principal conclusions obtained.

\section{KEY WORDS}

Prevention, Drugs, Young people, Music. 


\section{INTRODUCCIÓN}

El presente trabajo es fruto de una ya larga linea de intervención e investigación que tuvo como primer hito a destacar la publicación en la Revista Española de Drogodependencias (2002) y después en la Revista Adicciones (2004), de dos estudios que analizaban descriptivamente la relación entre letras de canciones españolas escuchadas por los jóvenes y tópicos temáticos relacionados con las drogas.

Esta investigación dio lugar a una aplicación práctica , estructurando el material analizado en un completo programa de prevención de drogodependencias para jóvenes escolares de $3^{0}$ y $4^{\circ}$ de la ESO y $1^{\circ}$ y $2^{\circ}$ de Bachillerato, que hemos denominado "Más musik. Prevenir en otra onda" y que consta de cinco sesiones en cada curso señalado.

Este programa se llevó a cabo como proyecto piloto financiado por la Agencia Antidroga de la Comunidad de Madrid $^{1}$, durante los meses de Marzo y Abril del 2004 en el Colegio Menesiano de Madrid, con unos resultados en general muy positivos, tanto en lo que se refiere a datos cuantitativos de diferencias significativas (por ejemplo, respecto al consumo de alcohol y cannabis antes y después del programa), como en datos cualitativos vinculados a la satisfacción con el programa así como la influencia atribuida a éste en la toma de decisiones futuras en cuanto al consumo de drogas 0 cambios en el consumo de sustancias . Más adelante tendremos ocasión de analizarlo con detalle.

\section{FUNDAMENTACIÓN}

El trabajo en prevención de drogas con los jóvenes es hoy un ámbito de especial interés entre otras cuestiones, por los datos epidemiológicos que presenta este grupo de edad. Sin embargo, está no es una tarea sencilla y nos encontramos con importantes problemas para desarrollarla. Además, podemos preguntarnos, en esta misma dirección, si esta todo inventado en materia de prevención o más bien estamos iniciando un largo trayecto.

También podemos cuestionarnos sobre la forma de conectar con los jóvenes y las necesarias explicaciones multi causales de este complejo fenómeno.

Por eso, para desarrollar "Prevenir en otra onda" partimos de un modelo consolidado, el modelo bio-psico-social y los factores de riesgo y protección asociados al mismo, en el que se contempla el consumo de sustancias desde la interacción de dimensiones, factores y variables psicológicas, sociales y biológicas o si lo preferimos: droga, persona y ambiente. La drogodependencia (tanto en lo que respecta a la adquisición como al mantenimiento y la extinción), el uso de sustancias o el abuso, son todos procesos complejos en el que la interacción entre múltiples factores es lo fundamental. Planteado así, el concepto clave es el uso que se haga de las drogas, que se convierte, por otra parte, en una construcción social, en una situación cultural, por lo que debemos contar con los sujetos para su conceptualización y comprensión.

Si se pretende, por tanto, desarrollar acciones de prevención con los jóvenes, hay que contactar con ellos. Como señala Funes (1996) hay que tener en cuenta el significado que para estos mismos jóvenes tienen estos consumos. Así, no

\footnotetext{
${ }^{1}$ Posteriormente, el programa ha sido financiado por el Plan Nacional Sobre drogas y aplicado a diferentes provincias y a nivel internacional, llegando a ser aplicado a más de 12.000 jóvenes.
} 
podemos caer en interpretar sus conductas bajo nuestro prisma; sino que, muy al contrario, de nuevo tendremos que verlo desde su punto de vista, por tanto, desde otra onda, desde su contexto y comunicarnos desde esta flexibilidad.

Siendo así, se nos presenta como fundamental conocer y manejar el mundo que vive el joven a quien vamos a dirigirnos.

En esta dirección, no hace falta explicar excesivamente el papel relevante que la música cumple, hoy por hoy, en la vida de la mayoría de la población joven (pero no solo de ésta). Viven su vida acompañados de música, convirtiéndose ésta en su banda sonora original. En esta linea, diferentes investigaciones, demuestran que, por ejemplo, dentro de los hábitos juveniles ocupan un lugar privilegiado algunos relacionados con la música (Martín Serrano, M., Velarde, O., 1997 I. Megias Quirós, E. Rodríguez; F.A.D e I.N.J.U.V.E. 2003). Así mismo, pueden constarse elevados gastos por parte de los jóvenes en música y de la industria musical en los jóvenes para promocionar sus productos. En otra dirección puede también señalarse el uso de la música para vender productos, como es el caso especifico de la industria tabaquera y de alcohol.

La música es, por tanto, un recurso importante, relevante, siginificativo para los jóvenes, un excelente instrumento a utilizar para establecer una longitud de onda preventiva diferente con los jóvenes, conectando con ellos de manera directa, clara y significativa, generando un clima de diálogo y trabajo bidireccional.

Se trata, pues, de utilizar la música para tratar de afrontar retos clásicos en materia de prevención de drogas con los jóvenes, cómo motivarles, desarrollar programas interactivos que cuenten con su participación, transmitirles información verídica y significativa, hablar de las consecuencias del uso y del abuso, de las motivaciones, de las estrategias de resistencia a la oferta, utilizando un lenguaje tan potente, significativo y motivador para los jóvenes como la música que escuchan o los mensajes que transmiten algunos de sus ídolos: los grupos y cantantes de música.

La música, no es solamente un elemento que contribuye al entretenimiento sino que, en muchos casos, sirve de vía para expresar los propios sentimientos, al mismo tiempo que los autores o interpretes de las melodias se convierten para el joven en modelos de conducta. La música es, igualmente, vehículo de transmisión de valores, creencias, actitudes y expectativas

Por otro lado, el trabajo con jóvenes puede hacerse desde la perspectiva de ellos mismos, facilitando así la adherencia a los programas, lo que dotaría a los mismos de una fiabilidad más elevada. Si los jóvenes se interesan por la música hablémosles a través de la música, pongámonos en su onda. Este estilo educativo garantiza al mismo tiempo una alta motivación y mayor facilidad para conectar con ellos, así como que ellos mismos se conviertan también en emisores de la información que poseen. Se convierten de esta manera en los protagonistas de los trabajos que con ellos realizamos. Se trata de, entre otras cuestiones, aumentar la interactividad de los programas mediante la participación activa de los usuarios y una mayor significación de los mensajes. No podemos hacer prevención para jóvenes sin la participación y los lenguajes de los propios jóvenes.

Uno de los principales problemas que se plantean en la actualidad respecto a la temática de las drogas es la baja percepción de riesgo asociado a los consumos. Los mensajes que llegan a nuestros jóve- 
nes son contradictorios. Por un lado, los adultos solemos reflejar una imagen basada en el miedo para evitar el consumo; por otra parte, los jóvenes transmiten información entre ellos de los afectos positivos que se producen con el consumo. Es innegable que estos efectos positivos existen y son los primeros en producirse (la hilaridad del cannabis, el aumento del bienestar de la cocaina, la desinhibición con el alcohol, por ejemplo) y son esos efectos los que los jóvenes buscan y por los que emplean las drogas. Estos efectos son los que más se manifiestan en los temas musicales lo cual refleja la realidad de los jóvenes y el reto de abordar la misma a corto y largo plazo.

Si prestamos un poco de atención (Fouce, 2002, 2004; investigación de la que parte el programa ya señalada anteriormente) a los mensajes que están contenidos en las canciones, encontramos que hay abundante información sobre drogas en cualquiera de los estilos musicales y que los mensajes que se lanzan, en general, tratan la temática con cierta frivolidad. Las drogas son algo divertido, que nos ayuda a pasarlo bien, a ligar, a salir con los amigos y también a olvidarnos de los problemas.

En general se construía en las letras musicales, una imagen que abogaba por el consumo recreativo, presentando las drogas como algo divertido. Eran pocos los casos en los que se hablaba de los efectos a medio y largo plazo que estas sustancias provocan en la salud y el bienestar de los que las utilizan.

Por todo esto, el proyecto "Música y prevención de drogas: Prevenir en otra onda”, se plantea como un cauce para aplicar la música a la prevención con jóvenes, como instrumento de trabajo y comunicación. La música como instrumento altamente eficaz para establecer una comunicación fiable con los jóvenes, aún por explorar y explotar (son muy pocas las fuentes que hemos encontrado en este sentido (entre las excepciones podemos encontrar los trabajos de Carles Sedo, Ray Parreño o la página http: //www.kolokon.com)

Partiendo de la música, interesándonos por lo que les gusta, nos acercamos a cada uno de los jóvenes y su pluralidad con sus mismos recursos de expresión, facilitando que una vez dentro de esta dinámica, sean ellos por sus propios medios los que descubran los mensajes, criben las ideas y tomen sus propias decisiones. Todo ello, contando con que poseen información significativa, cercana y relevante.

Además, durante esta etapa evolutiva de la adolescencia y juventud, la música también puede convertirse en un elemento identificador, tanto de la individualidad de cada uno como de la pertenencia al grupo.

Otra incidencia de la música y su utilización preventiva puede encontrarse en la fractura del tiempo cronológico en dos en la vida de los jóvenes: por un lado el tiempo de trabajo o estudio, entendido como tiempo normativizado, rutinizado; por otro lado, el tiempo de fiesta; "el tiempo de trabajo o estudio a la postre no sería sino un tiempo por el que hay que pasar lo más rápidamente posible para disfrutar del tiempo de fiesta que se convierte así en "el tiempo" por antonomasia” (Elzo, 1994:16). Con frecuencia, se da la paradoja, sin embargo, de que los programas preventivos destinados a jóvenes se insertan en su tiempo de estudio o trabajo, utilizando elementos de este contexto pero tratándose de incidir en el otro tiempo fracturado, el tiempo de ocio, el tiempo de la fiesta y la expresión con lo cual, tienen dificil establecer conexiones significativas entre estos dos tiempos. 


\section{FUNDAMENTOS DE PREVENCION DE PREVENIR EN OTRA ONDA}

La prevención de drogas es hoy un campo prioritario para las administraciones considerándose, especialmente, la necesidad de desarrollar proyectos de prevención evaluables y evaluados que demuestren conseguir los objetivos perseguidos. En este énfasis en la prevención se han ido resaltando algunos elementos básicos fundamentales a la hora de diseñar y ejecutar programas de prevención. Entre estos elementos, por su especial relevancia para la intervención que proponemos queremos destacar los siguientes (Becoña, 2002; Amador y Calafat, 1999):

- Los programas preventivos deberían ser diseñados para realzar los factores de protección y revertir o reducir los factores de riesgo. Los programas preventivos deberian adaptarse a los conocimientos u percepciones de los destinatarios.

- Los programas preventivos deberían incluir todas las formas de consumo de drogas, incluyendo tabaco, alcohol, marihuana e inhalantes.

- Los programas preventivos para los adolescentes deberían incluir métodos interactivos obligando a los jóvenes a participar en lugar de solo técnicas didácticas. Evitar confiar únicamente en las intervenciones basadas en los conocimientos diseñadas para proporcionar información sobre las consecuencias negativas del consumo.

- Los programas preventivos deberían ser a largo plazo, a lo largo de los años escolares con intervenciones repetidas para reforzar las metas preventivas originales.
- La escuela ofrece la posibilidad de alcanzar a toda la población de jóvenes y también sirve como un lugar importante para sub poblaciones específicas con mayor riesgo de consumir drogas.

- Es efectivo enseñar habilidades para la vida genéricas como toma de decisiones, habilidades de resistencia a influencias de los otros, habilidades sociales, en reducir la incidencia del abuso de sustancias entre los jóvenes.

- Implicar a los jóvenes en intervenciones con iguales o con intervenciones que tienen componentes que utilizan iguales.

- Hay que proporcionar a los estudiantes oportunidades para practicar las nuevas habilidades adquiridas a través de aproximaciones interactivas.

\section{DESARROLLO DEL PROGRAMA PREVENIR EN OTRA ONDA}

\subsection{Objetivos}

1. Desarrollar un programa interactivo en el que los jóvenes participen activamente desde el principio del programa hasta el final del mismo.

2. Aumentar el interés y la significación de los contenidos preventivos.

3. Aumentar la cercanía y significatividad del programa así como su carácter lúdico- formativo.

4. Aumentar en la credibilidad de la información transmitida (Utilizar lenguaje cercano). 
5. Tratar de llegar a grupos diferentes de jóvenes con motivaciones, realidades, estilos de vida y lenguajes diferentes

\subsection{Actividades}

El programa "Prevenir en otra onda" está secuenciado para su aplicación en los cuatro últimos cursos escolares, aumentando la profundidad en que se tratan los diferentes temas y las habilidades necesarias para las diferentes actividades, con la edad de los jóvenes a los que está destinado, en función de los datos existentes en la actualidad de prevalencia de consumo de sustancias según las edades.

El esquema general de aplicación es el mismo para todos los cursos, pero van cambiando los recursos utilizados, la metodología o dinámicas empleadas y las drogas que se trabajan. Así, bajo los mismos títulos de las sesiones se encuentran dinámicas diferentes para cada edad, sustancias distintas tratadas de maneras similares o metodologias que van evolucionando en los distintos cursos. De está manera se consigue que el mismo joven pueda insertarse de forma repetida en el programa (en los diferentes cursos) sin que lleguen a agotarse los recursos que este ofrece y sin que los alumnos lleguen a aburrirse.

Esa es una de las grandes ventajas que tiene la utilización de la música para interactuar con los escolares. Permite una gran variedad de tratamientos, una ampliación constante del material, así como tareas siempre innovadoras y diferentes. Y dado que es el lenguaje del joven, permite que éste se encuentre muy pronto adaptado a la metodología y la disfrute desde el principio. Así las cosas, lo que el programa busca es que se complete la información que el joven recibe desde esta vía sobre la temática de drogas, con información precisa y completa y con formación que le permita evolucionar a nivel personal.

Para el primer curso ( $3^{\circ}$ de E.S.O.), el programa consiste en una visión de las diferentes sustancias; mientras, en los cursos siguientes se tratan con mas profundidad sustancias concretas: en $4^{-}$de E.S.O, alcohol y tabaco; en $1^{\circ}$ de Bachillerato, cannabis y en $2^{\circ}$ de Bachillerato, la apuesta es hablar de drogas como la cocaina o las drogas de sintesis.

\subsection{Metodologia}

A continuación se presenta la tabla resumen de las diferentes sesiones. En ellas se pueden encontrar los objetivos de las sesiones, la temática de la que se va a tratar en cada una de ellas y las metodologias empleadas.

Quizá, cabria destacar entre las novedades de la metodología y sesiones planificadas (además de la utilización de la música para potenciar la interactividad del programa o la participación de los jóvenes como ya se menciono anteriormente) el desarrollo de una última sesión centrada en la dimensión comunitaria del programa, tratando de ir más allá de las sesiones establecidas y también de comprometer personal y públicamente a los participantes en la temática y de conectar los diferentes ámbitos de prevención. También se opta, como puede comprobarse, por la ejecución práctica de habilidades como la resistencia a la presión o la transmisión de información normativa significativa de manera motivacionalmente relevante o se pasa de la reflexión y trabajo individual a lo colectivo. 
Tabla 1. Reumen de sesiones de prevenir en otra onda

\begin{tabular}{|c|c|c|}
\hline Temática & Objetivos & Metodologias \\
\hline $\begin{array}{l}\text { 1. ¿En qué onda éstas? } \\
\text { Música y drogas. Drogas en el mundo de } \\
\text { la música. } \\
\text { ¿Qué hacen ELLOS? }\end{array}$ & $\begin{array}{l}\text { - Identificar los gustos y preferencias } \\
\text { musicales de adolescentes y jóvenes con el } \\
\text { fin de actuar con creatividad. } \\
\text { - Evaluar su situación de partida con } \\
\text { respecto a hábitos de consumo y hábitos } \\
\text { de disfrute del tiempo libre. } \\
\text { - Potenciar la conexión con el programa. } \\
\text { - Fomentar la motivación con el programa. } \\
\text { - Potenciar la interactividad y la } \\
\text { significatividad del program. Que los } \\
\text { adolescentes y jóvenes tomen conciencia } \\
\text { de los valores y actitudes que pueden } \\
\text { llevar de forma intrínseca el mundo de la } \\
\text { música. } \\
\text { - Analizar diferencias en los gustos } \\
\text { musicales y en niveles de relación con las } \\
\text { - Tustancias. } \\
\text { no consunsumo. }\end{array}$ & $\begin{array}{l}\text { - Sesión introductoria. } \\
\text { - Presentación de los integrantes del grupo } \\
\text { y del educador. } \\
\text { - Recogida de información de características } \\
\text { individuales y grupales: gustos musicales, } \\
\text { consumo de tiempo de ocio. } \\
\text { - Dinámica motivadora: audición de } \\
\text { canciones según sus gustos. } \\
\text { - Puesta en común de la información que } \\
\text { aportan los integrantes del grupo. } \\
\text { - Análisis de las letras y autores o grupos. } \\
\text { - Audición de una canción. } \\
\text { - Debate: contraste con la información } \\
\text { aportada por el educador. }\end{array}$ \\
\hline $\begin{array}{l}\text { 2. ¿Qué sabes de las drogas? } \\
\text { - Conceptos básicos. } \\
\text { - Mitos y realidades. } \\
\text { - Situación legal. }\end{array}$ & $\begin{array}{l}\text { - Identificar las creencias que tienen } \\
\text { adolescentes y jóvenes a cerca del } \\
\text { consumo de drogas. } \\
\text { - Que los alumnos reflexionen sobre las } \\
\text { opiniones, creencias, actitudes y valores } \\
\text { relacionados con el consumo de drogas. } \\
\text { - Conocer las consecuencias legales } \\
\text { asociadas al consumo, posesión y tráfico } \\
\text { de drogas. }\end{array}$ & $\begin{array}{l}\text { - Lluvia de ideas. } \\
\text { - Juego "Prevenir en otra onda". } \\
\text { - Ensayo de conducta sobre oposición a } \\
\text { presión grupal mediante asertividad. }\end{array}$ \\
\hline $\begin{array}{l}\text { 3. Aquel día que me pasé. } \\
\text { - Consecuencia del consumo de drogas. } \\
\text { - Conductas de riesgo asociadas. } \\
\text { - Accidentes de tráfico. } \\
\text { - Relaciones sexuales. } \\
\text { - Violencia. } \\
\text { - ¿Merece la pena así? } \\
\text { - Motivaciones para el consumo. } \\
\text { - Ocio alternativo. }\end{array}$ & $\begin{array}{l}\text { - Fomentar en la población adolescente y } \\
\text { juvenil una toma de conciencia realista } \\
\text { sobre los riesgos asociados al consumo } \\
\text { de drogas. } \\
\text { - Reflexionar sobre los riesgos asociados al } \\
\text { consumo de drogas. } \\
\text { - Orientar a los adolescentes y jóvenes } \\
\text { sobre las conductas de riesgo ligados al } \\
\text { consumo de drogas. Transmitir } \\
\text { información que limite los posibles } \\
\text { riesgos. } \\
\text { - Definir consecuencias negativas } \\
\text { inmediatas y cercanas al joven. } \\
\text { - Promover una actitud critica en } \\
\text { adolescentes y jóvenes frente a los } \\
\text { motivos. } \\
\text { - Dar a conocer a los adolescentes y } \\
\text { jóvenes las alternativas de ocio } \\
\text { existentes. }\end{array}$ & $\begin{array}{l}\text { - Dinámica de formación de subrupos } \\
\text { - según gustos musicales. } \\
\text { - Audiciôn de las canciones adecuadas al } \\
\text { tema. } \\
\text { - Análisis de las letras. } \\
\text { - Recogida de las conclusiones del } \\
\text { análisis. } \\
\text { - Entrega de tabla sobre los efectos de las } \\
\text { diferentes drogas a corto, medio y largo } \\
\text { plazo. } \\
\text { - Historias personales. } \\
\text { - Entrega de listín telefónico de asistencia. } \\
\text { - Role playing de situaciones en las que se } \\
\text { suele consumir. } \\
\text { - Ensayo de conductas alternativas al } \\
\text { consumo. }\end{array}$ \\
\hline 4. ¿Qué pasa CONMIGO? & $\begin{array}{l}\text { - Reforzar estilos de vida saludables. } \\
\text { - Trabajar variables individuales: } \\
\text { autoestima, autocontrol. } \\
\text { - Trabajar variables de carácter grupal: } \\
\text { participación, relaciones sociales, } \\
\text { respeto, comunicación, actitud critica. } \\
\text { - Fomentar una actitud positiva ante la } \\
\text { vida. } \\
\text { - Crear positivamente las actuaciones } \\
\text { desarrolladas. }\end{array}$ & $\begin{array}{l}\text { - Dinámica de grupo para dividir en } \\
\text { subgrupos según estilos musicales. } \\
\text { - Trabajo cooperativo de elección de una } \\
\text { camcoón para cada estilo. } \\
\text { - Presentación plástica de estilos de vida } \\
\text { positivos y negativos. }\end{array}$ \\
\hline 5. Y nosotros, ¿qué podemos hacer? & $\begin{array}{l}\text { - Transmitir modelos positivos asociados } \\
\text { al consumo. } \\
\text { - Proporcionar oportunidades prácticas de } \\
\text { ejercicio de lo aprendido o transmitido en } \\
\text { el programa }\end{array}$ & $\begin{array}{l}\text { - Karaoke. } \\
\text { - Video-clip. } \\
\text { - Coreografia. } \\
\text { - Discoteca light. } \\
\text { - Dinámica de despedida. }\end{array}$ \\
\hline
\end{tabular}




\section{EVALUACIÓN}

\section{a) Diseño}

En el desarrollo del programa, por ser esta la primera aplicación del mismo y, por tanto, tener una consideración de proyecto piloto, se tuvo un especial cuidado en el diseño de un proceso evaluativo que permitiese establecer conclusiones sólidas y validadas empíricamente una vez terminada la ejecución del programa.

Como se señala hoy desde todos los parámetros técnicos, y reconoce entre otros documentos la Estrategia Nacional sobre drogas 2000- 2008 o en el documento de "criterios básicos de intervención de los programas de prevención de drogodependencias" necesitamos construir programas y acciones de prevención debidamente evaluados mediante métodos científicos rigurosos y fiables que nos permitan afirmar claramente si la acción preventiva que estamos desarrollando tiene 0 no un efecto preventivo, sobre que variables o factores y en que grado o medida.

Así, diseñamos una triple estrategia evaluativa:

- En primer lugar, se estableció una medida previa al desarrollo del programa tanto con los grupos experimentales como con el grupo control. Esta medida sirve de línea base para establecer posteriores comparaciones. Se comparará con los datos de los que disponemos hasta el momento de las encuestas escolares desarrolladas por el Plan Nacional de Drogas a lo largo del tiempo para constatar la situación de nuestra muestra con respecto al conjunto extenso de población juvenil de la Comunidad de Madrid y el Estado.
- En segundo lugar, se estableció un diseño con grupo control y grupo experimental que recibieron un tratamiento experimental diferente (recibir el programa versus no recibir el programa) y que posteriormente fueron evaluados con respecto a un mismo cuestionario que, a su vez, conectaba con la ya señalada medida previa para establecer una doble comparación entre grupo control y grupo experimental y entre medias pre y post.

- En tercer lugar, se desarrolló una evaluación interna consistente en el desarrollo de un cuestionario valorativo (cuantitativo y cualitativo) al final de la ejecución del programa piloto con los grupos sometidos a tratamiento experimental (desarrollo del programa). En este cuestionario se evalúo la satisfacción de los participantes con el programa, el grado de aprendizaje y las actitudes generadas, las posibles mejoras que sugieren los participantes y los aspectos que más satisfacción- insatisfacción causaron en los jóvenes.

Entre las variables que se tomaron en consideración encontramos:

a) Variables contextualizadoras y sociodemográficas.

b) Relación con respecto al consumo de diferentes drogas.

- Variables preventivas (Nivel de información con respecto a las drogas y utilidad percibida de esta información; Percepción del nivel de consumo de alcohol con respecto a la media; Intención de dejar de fumar e intentos desarrollados para dejar de fumar, etc.).

c) Satisfacción con el programa, valo- 
ración del mismo, aportaciones y déficit. (en el cuestionario interno).

La variable que determinaba la condición experimental del diseño era la aplicación 0 no del programa de prevención "Prevenir en otra Onda" en cada uno de las aulas en las que el mismo se ejecutó.

Se pretende por tanto, establecer por una parte, la posible incidencia del programa en las variables mencionadas y por otra parte, la valía intrínseca del mismo según las aportaciones desarrolladas por los jóvenes que participaron en el mismo (grupo experimental).

\section{b) Sujetos- muestra}

Los jóvenes alumnos participantes en la experiencia piloto, son todos alumnos del centro concertado Colegio Menesiano (Madrid) seleccionado para el desarrollo de este pilotaje por diferentes cuestiones operativas entre las que cabe mencionar la especial disposición de la dirección del colegio y su claustro de profesores a participar en una experiencia de este tipo.

El número total de alumnos analizados fue de 160 de ellos 85 pertenecian al grupo control y 75 al grupo experimental con la siguiente distribución:

La elección entre alumnos participantes en el grupo experimental o en el grupo control se hizo de manera aleatoria a partir de la distribución de los jóve- nes por cursos escolares de los alumnos. Estando configuradas las clases, así mismo, mediante un criterio aleatorio como son los apellidos de los alumnos, lo cual garantiza en todo momento la ausencia de sesgos producidos por la falta de aleatoriedad en el proceso desarrollado.

\section{c) Instrumentos de medida utilizados:}

Se emplearon tres cuestionarios para establecer la medida de las variables anteriormente señaladas: cuestionario pre (antes de la ejecución del programa), un cuestionario post (tras la ejecución del programa, coincidiendo su aplicación en el mismo momento temporal entre el grupo pre y el post) y un cuestionario de evaluación interna (valoración cualitativa y cuantitativa del programa por los participantes en el mismo).

Para la elaboración de los dos primeros cuestionarios se contó con preguntas ya suficientemente validadas y que permitieran una comparación con otros estudios anteriores. Estas preguntas se extraen de la Encuesta Escolar sobre consumo de drogas desarrollada en los últimos años secuencialmente por el Plan Nacional de Drogas. Algunas de las preguntas fueron modificadas, si bien mantenemos la esencia de las mismas.

Entre las modificaciones, se encuentra la transformación con respecto a la percepción de riesgo ligadas al consumo

Tabla 2. Sujetos participantes

\begin{tabular}{|l|c|c|c|c|c|}
\hline & $3 \mathrm{ESO}$ & $4 \mathrm{ESO}$ & $1 \mathrm{BACH}$ & $2 \mathrm{BACH}$ & TOTALES \\
\hline G. Control & 23 & 19 & 25 & 18 & 85 \\
\hline G. Experimental & 9 & 10 & 23 & 33 & 75 \\
\hline & 32 & 29 & 48 & 51 & 160 \\
\hline
\end{tabular}


de drogas que se encuentra en este cuestionario, en una dimensión escalar centrada en cada una de las sustancias trabajadas en el programa.

Estos dos primeros cuestionarios utilizados (pre y post) tienen una estructura de preguntas similar si bien el cuestionario post, para evitar sesgos contaminadores producidos por la reiteración de las preguntas, tiene aleatorizadas las preguntas sobre dimensiones claves consideradas como la percepción de riesgo 0 las intenciones de consumo. Así mismo, cabe señalar que, si bien los cuestionarios eran anónimos en todo momento, se codificaban de acuerdo a la posición que los alumnos ocupaban en sus respectivas clases, posición que se mantenía entre el primer momento y el segundo momento de exploración, lo que permitía ahorrarse preguntas de carácter contextualizador y sociodemográficas entre el cuestionario pre y el cuestionario post; así como ahorrar tiempos y costes y permitir presentar un cuestionario post diferente al pre en su forma y formato evitando así, elementos distorsionadores o de sesgo de respuesta por habituación en los jóvenes que contestan al mismo.

El tercer cuestionario, el cuestionario de evaluación de carácter interno, se diseño ad hoc para el desarrollo del presente programa constituyéndose en una parte intrínseca del mismo como parte del material a utilizar en posteriores y futuras aplicaciones del programa. Para el desarrollo de este cuestionario se combinaron preguntas cerradas con abiertas de manera que pudiésemos disponer de información cuantitativa y cualitativa sobre el desarrollo del programa, la satisfacción con el mismo y las aportaciones sugeridas por los participantes.

Entre los aspectos considerados en este cuestionario de evaluación interno, se consideran elementos como las valo- raciones cuantitativas que los participantes desarrollan sobre cada una de las sesiones en las que participan, la valoración que hacen de la actuación de la monitor, la influencia atribuida al programa en la toma de decisiones con respecto al consumo o relación con las drogas, las mejoras que sugieren, los aspectos más destacados y los menos destacados, y las sugerencias que plantean al desarrollo.

\section{DESCRIPCIÓN DE LOS RESULTADOS}

\section{A. Datos cualitativos}

El cuestionario interno nos permitió conocer las opiniones de los alumnos en cuanto a las diferentes sesiones. A continuación se presentan los resultados de obtenidos en los diferentes grupos en cuanto al grado de satisfacción de los alumnos con las sesiones, el programa en general y el trabajo de la monitora:

Los resultados de estos gráficos muestran como las sesiones del programa "Prevenir en otra onda", son bastante interesantes para los alumnos, siendo la puntuación más alta obtenida(en cuanto a las sesiones) por la quinta sesión para $4^{0}$ de la E.S.O., en la que los alumnos eran los responsables de llevar a cabo una discoteca light (sin alcohol) y se desarrollaba una actividad de cócteles sin alcohol; la puntuación más baja es para la primera sesión para $2^{\circ}$ de Bachillerato, en las que los alumnos tenian que analizar algunas canciones de diferentes estilos propuestas por el monitor y ajenas a la temática que nos ocupaba. La media respecto a la satisfacción con el programa se sitúa en 3'35 (bastante interesante) y la del trabajo de la monitora en 3,5 (idem).

En los siguientes gráficos se muestran los resultados de la pregunta número 
Grafico 1. Indices de satisfacción en $3^{\circ}$ de E.S.O.

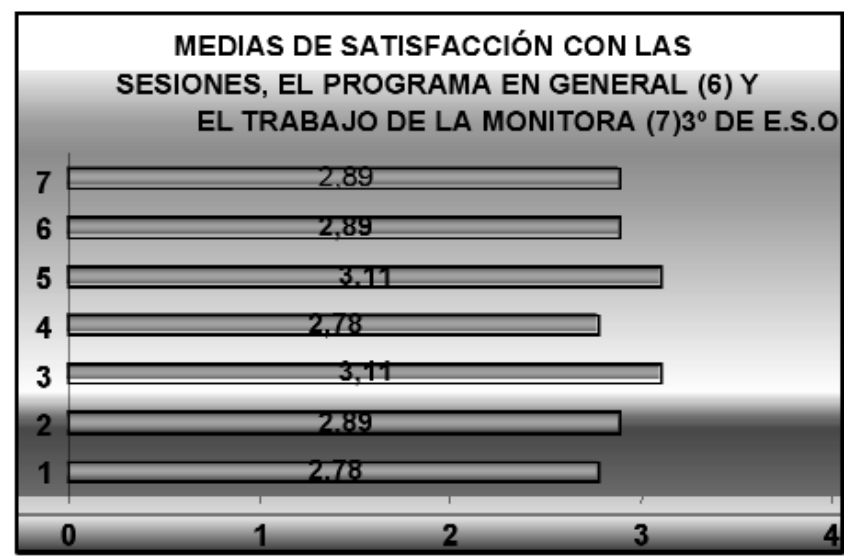

Grafico 2. Indices de satisfaccion en $4^{\circ}$ de la E.S.O.

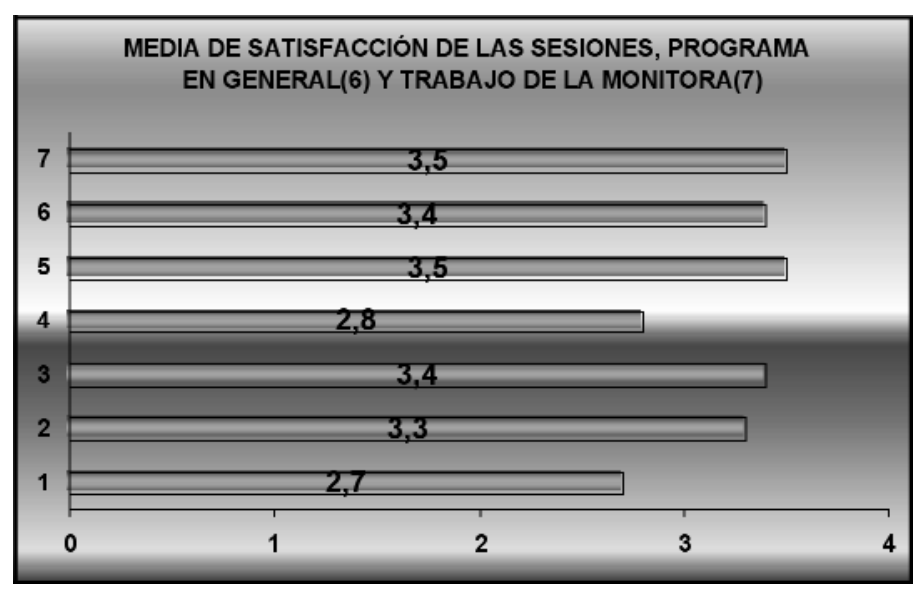

ocho (" $¿ H a$ influido el programa en que tomes alguna decisión respecto al consumo de drogas? En caso afirmativo, ¿qué decisión?"), a las que se suma una tabla resumen (Grafico 9) en la que se engloban las anteriores (Graficos 5,6, 7 y 8).

Como puede observarse, en todos los casos se reconoce una influencia del programa superior al 25\% (para $1^{\circ}$ de Bachillerato), llegando en el caso de $3^{\circ}$ de E.S.O. al 56\% (tabla 5). A partir de estos datos, se elabora el gráfico siguiente, con las medias de los alumnos de todos los cursos. El $40 \%$ de los alumnos que han tenido la experiencia de "Prevenir en otra onda" dice que el programa influye en su decisión sobre las drogas y todo parece indicar que la tendencia es hacia el no consumo. Del $60 \%$ que dice que esta influencia no es para la decisión, un 50\% aproximadamente dice tener ya tomada esa decisión y que el programa ha contribuido a ratificarla (en la misma dirección que lo anterior). 


\section{Grafico3. Indices de satisfacción en $1^{\circ}$ de Bachillerato}

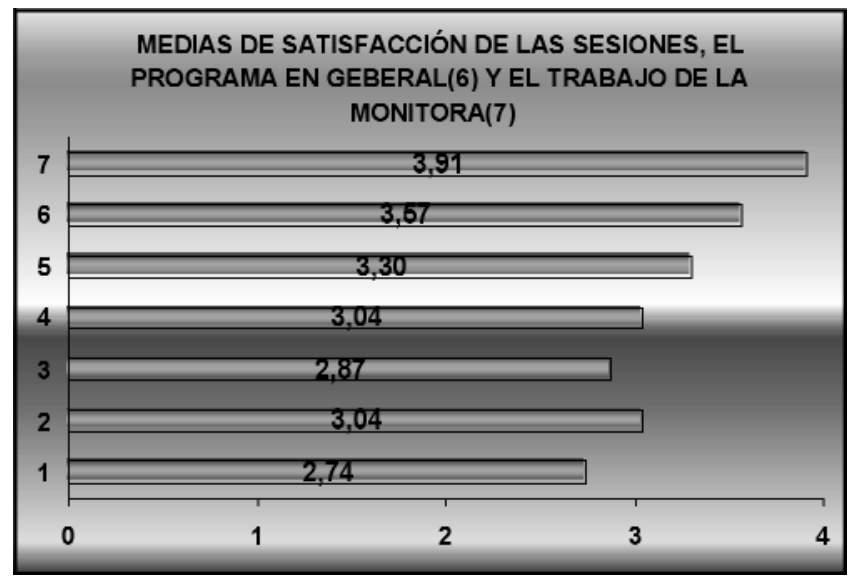

Grafico 4. Indices de satisfacción en $2^{\circ}$ de Bachillerato

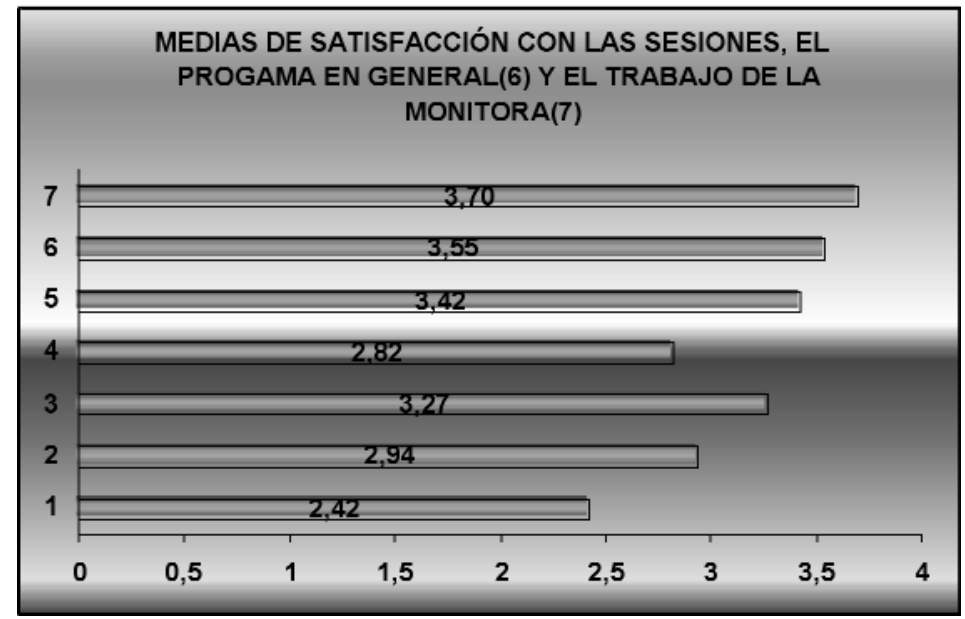

A continuación se presentan algunas de las razones que dan los alumnos para esta influencia. Según los grupos de edad se valoran en mayor o menor medida diferentes aspectos. Para los más jóvenes ( $3^{\circ}$ de E.S.O.; grafico 10 ), la información que aporta el programa es lo que más influye a la hora de tomar decisiones. En cambio, los alumnos de $4^{\circ}$ de la E.S.O., valoran más el fomento de sus habilidades personales, lo que es común a los alumnos mayores(gráfico 13). Para los de $1^{0}$ de Bachillerato ha influido a través de la promoción de habilidades personales y en este caso desaparece la categoría "información".

Las dos preguntas siguientes eran de tipo abierto e indagaban en los aspectos, temáticas, dinámicas, etc., que más y menos les habían gustado y en las razo- 
Grafico 5. Influencia del programa en $3^{\circ}$ ESO

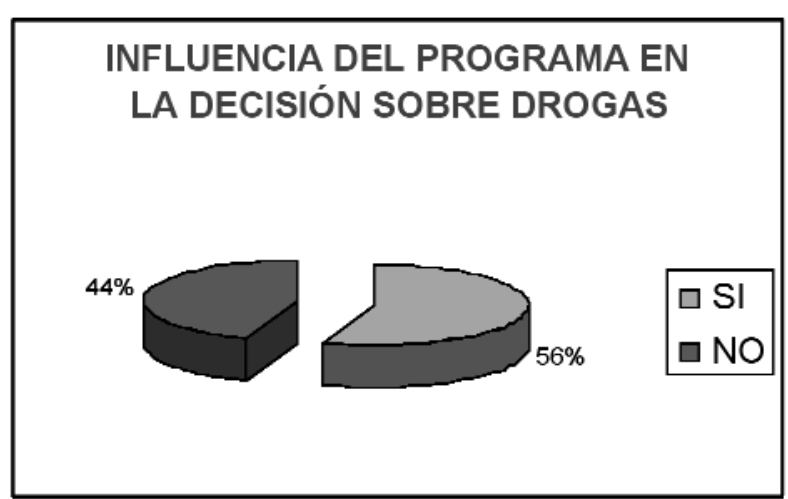

Grafico 6. Influencia del programa en $4^{\circ}$ ESO

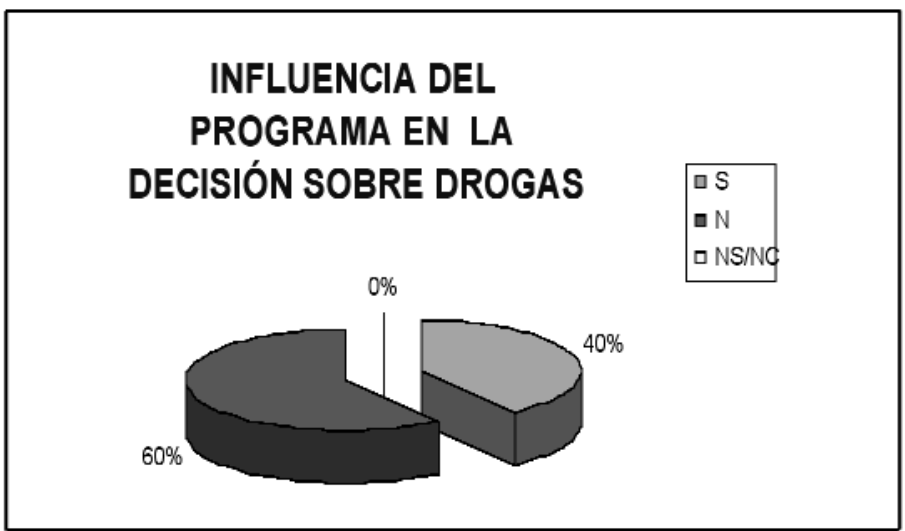

nes de su elección. En general las respuestas dirigen nuestra atención a la información que el programa aporta, así como sobre la posibilidad de compartir experiencias personales y de resolver sus dudas que la dinámica permite. Para ellos lo más valorado es que sea interesante, por la información que aporta, así como poder compartir con sus compañeros. Llama la atención que en todos los grupos se den respuestas generales como "Todo" y que el trato con la monitora salga a relucir. Es una de las características que permite "Prevenir en otra onda".
Cabe señalar que las categorías cambian en los diferentes cursos por estar tomadas las respuestas literalmente de los cuestionarios de los alumnos.

En cuanto a los aspectos que menos gustaron, la mayoría de los adolescentes de opinan que no hay nada que no les guste. Las diferencias entre los grupos son evidentes en este apartado por las diferentes dinámicas llevadas a cabo con cada uno de ellos. Entre las dinámicas mencionadas por los alumnos, el juego de "Prevenir en otra onda" sale en todos los cursos, aunque son minoritarias 
Grafico 7. Influencia del programa en $1^{\circ}$ Bachillerato

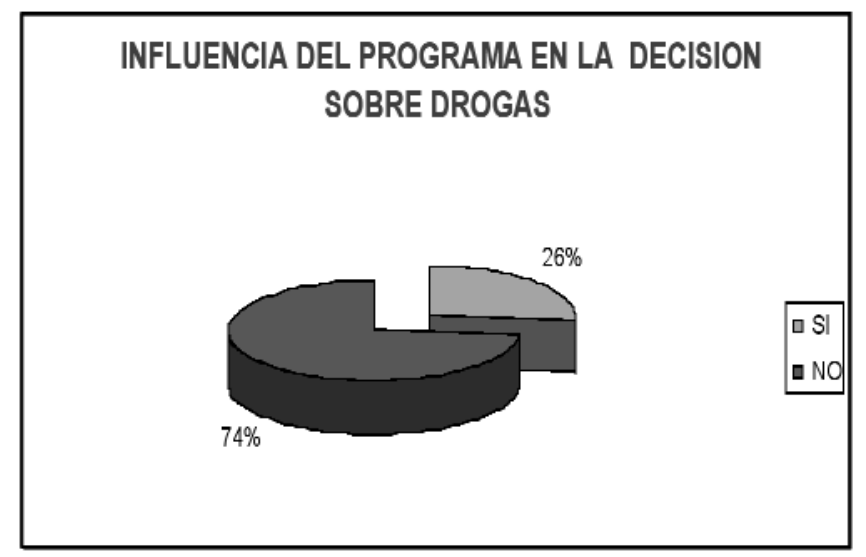

Grafico 8. Influencia del programa en $2^{\circ}$ Bachillerato

\section{INFLUENCIA DEL PROGRAMA EN LA TOMA DE DECISIONES SOBRE DROGAS}
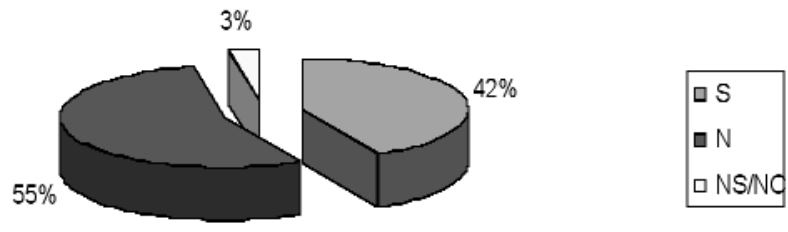

estas opiniones. Por último, algunos mencionan como crítica que hay trabajar para participar en el programa (pensar para realizar algunas de las actividades y dinámicas, escribir, etc.).

También nos interesaba averiguar hasta que punto el programa enseñaba algo, o dicho de otra manera, que aprendian los alumnos con los que trabajamos. Para obtener respuesta se incluyó en el cuestionario interno una pregunta en la que ellos podían decirnos tres cosas que hubieran aprendido en el taller. En los gráficos de los gráficos 14, $15,16,17$ se muestran sus respuestas de los cuatro grupos en diferentes categorías.

La categoría información en la que se englobaron para el análisis de los datos diferentes tipos de respuestas, se menciona en todos los grupos, así como la de consecuencias negativas. Como ocurre en el caso anterior, las diferencias entre los grupos vienen explicadas por las dife- 
Tabla 9. Influencia del programa resumen todos los cursos

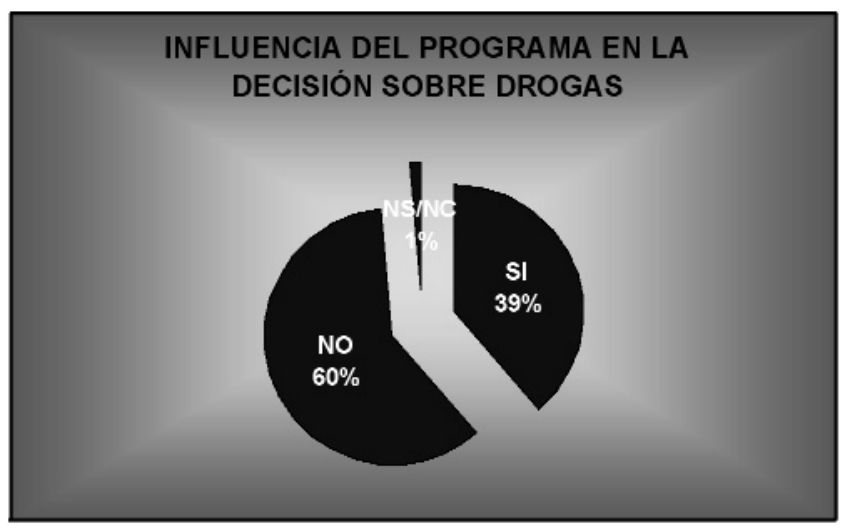

Grafico 10:. Razones de influencia $3^{\circ}$ E.S.O.

RAZONES POR LAS QUE EL PROGRAMA INFLUYE

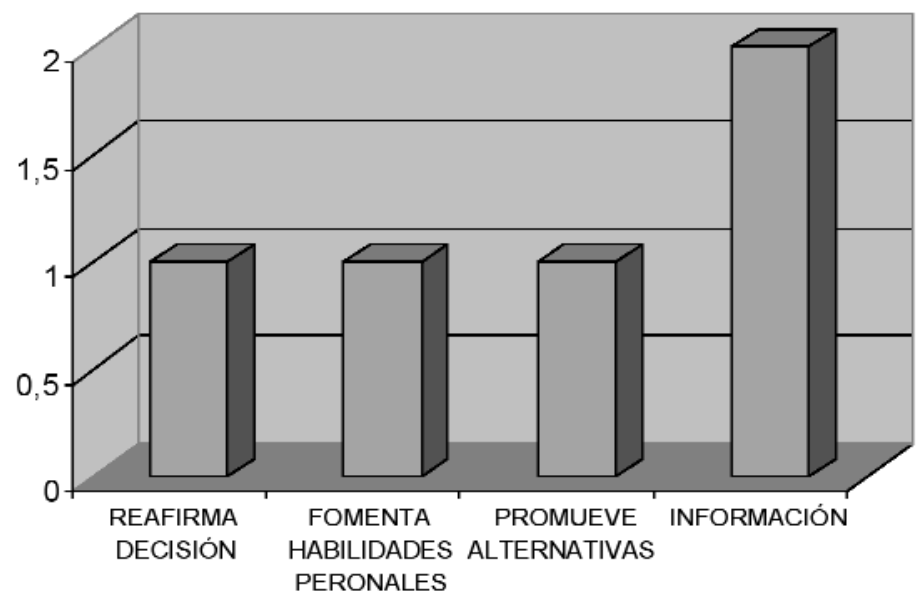

rentes dinámicas realizadas en las aulas. Así en el gráfico 15 se menciona como aprendizaje la elaboración de cócteles sin alcohol, al igual que se mencionan específicamente las consecuencias negativas del consumo de las drogas específicas trabajadas en este grupo: alcohol y tabaco.

La relación entre música y drogas, es decir, que en la música actual se refleja información sobre esta temática, tam- bién se ve reflejadas en los grupos de mayores (graficos 16 y 17).

Los últimos gráficos elaborados para las respuestas de los grupos, reflejan los resultados de la pregunta número doce sobre las cosas que ellos cambiarían o mejorarian. La mayoría señala que no cambiaría nada. Las otras categorías varían de un grupo a otro por las mismas razones mencionadas anteriormente. Algunos opinan que deberían 
Grafico 11. Razones de influencia 4 ESO RAZONES POR LAS QUE EL PROGRAMA INFLUYE

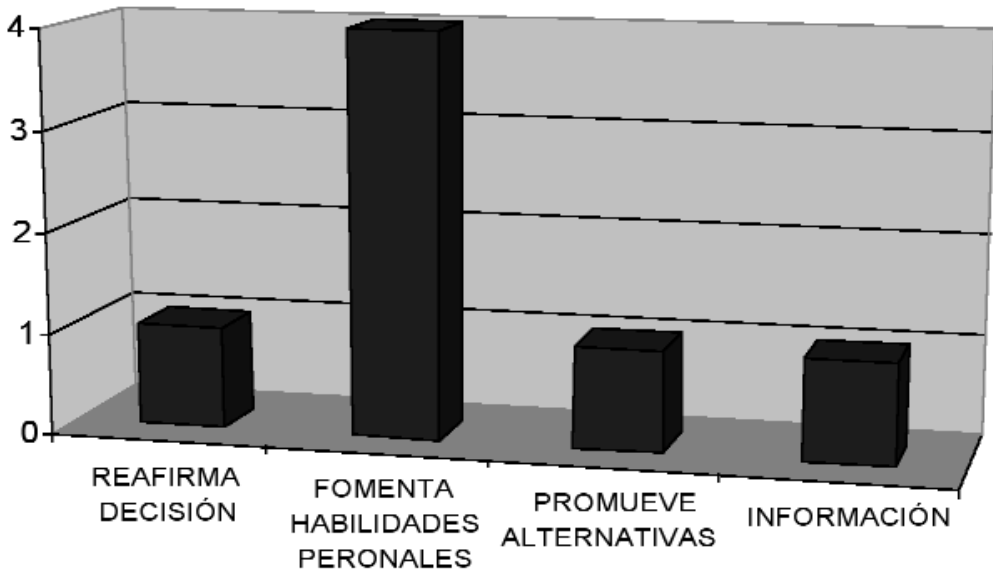

Grafico 12. Razones de influencia $1^{\circ}$ BACHILLERATO RAZONES POR LAS QUE EL PROGRAMA INFLUYE

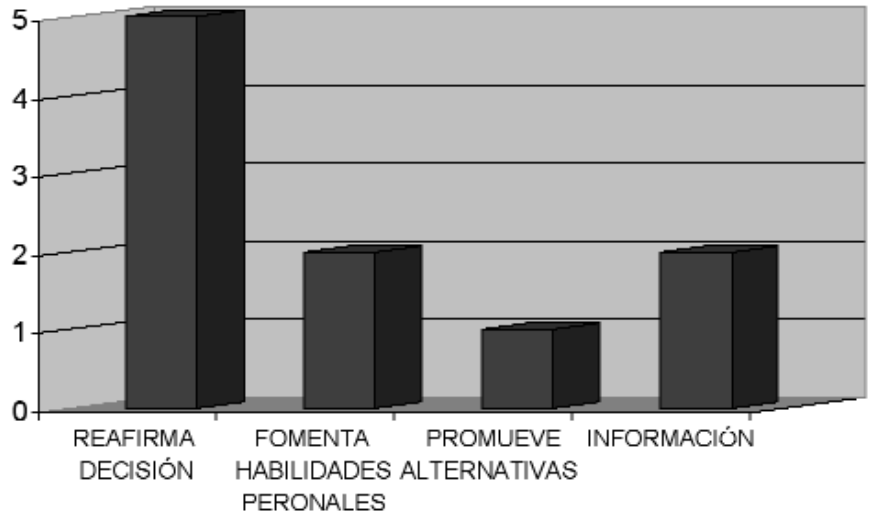

incluirse más experiencias personales; otros aumentarían la duración del programa.

\section{B. Datos cuantitativos}

A continuación se presentan los resul- tados estadísticos obtenidos a partir de las pruebas realizadas con los cuestionarios desarrollados para el pilotaje antes y después de desarrollar la acción preventiva y comparando entre grupo control y experimental. En ellas pueden apreciarse los cambios que hay entre la condición pre y la condición post, cambios atribui- 
Grafico 13, Razones de influencia $2^{\circ}$ RAZONES POR LAS QUE EL PROGRAMA INFLUYE

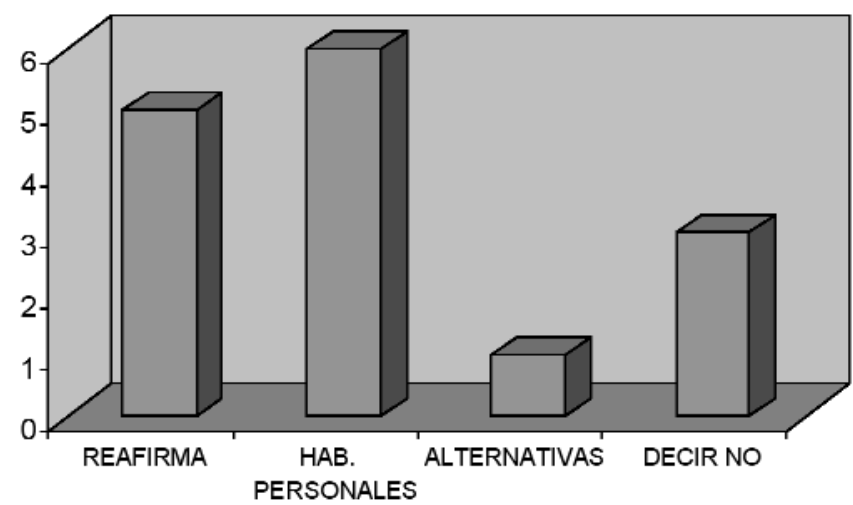

Gráfico 14. Aprendizajes en $3^{\circ}$

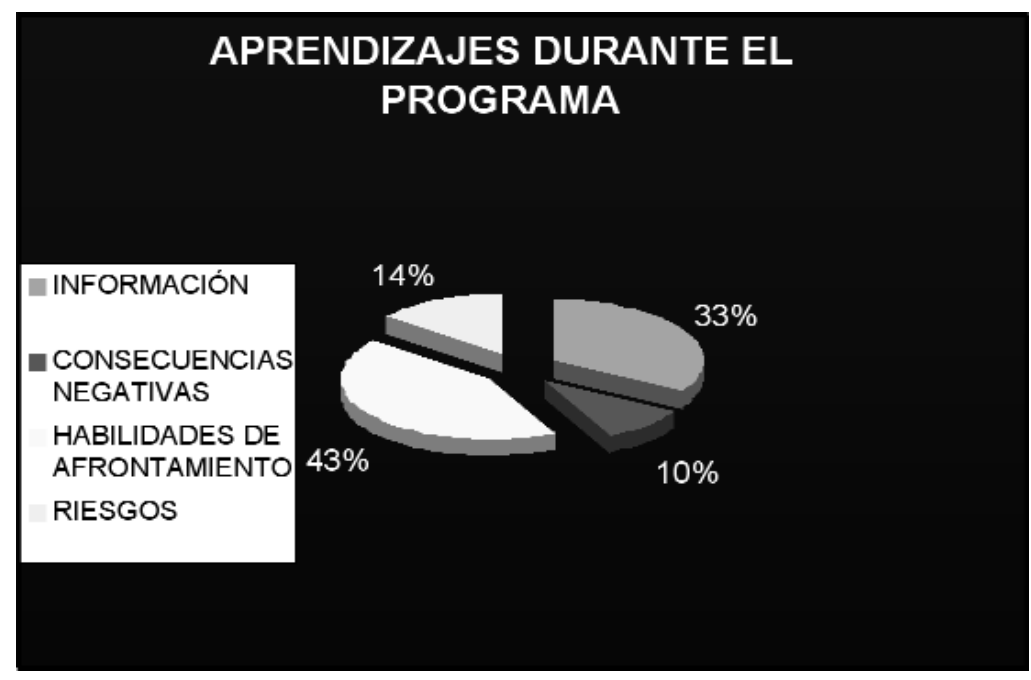

bles, por tanto a la ejecución de "Prevenir en otra onda".

Para este análisis nos hemos centrado en algunas variables ya descritas, como son: el consumo de drogas legales (alcohol y tabaco) e ilegales (cannabis), las cantidades de ingesta de estas sustancias (incluyendo para el alcohol el consu- mo agudo), el grado en que los jóvenes se sienten informados y la utilidad de esta información, etc.

En cuanto las variables en las que se han encontrado cambios estadísticamente significativos, a partir del desarrollo de correlaciones, la primera que encontramos es la intención de dejar de fumar. 
Gráfico 15. Aprendizajes en $4^{\circ}$ ESO

\section{APRENDIZAJES DURANTE EL PROGRMA}
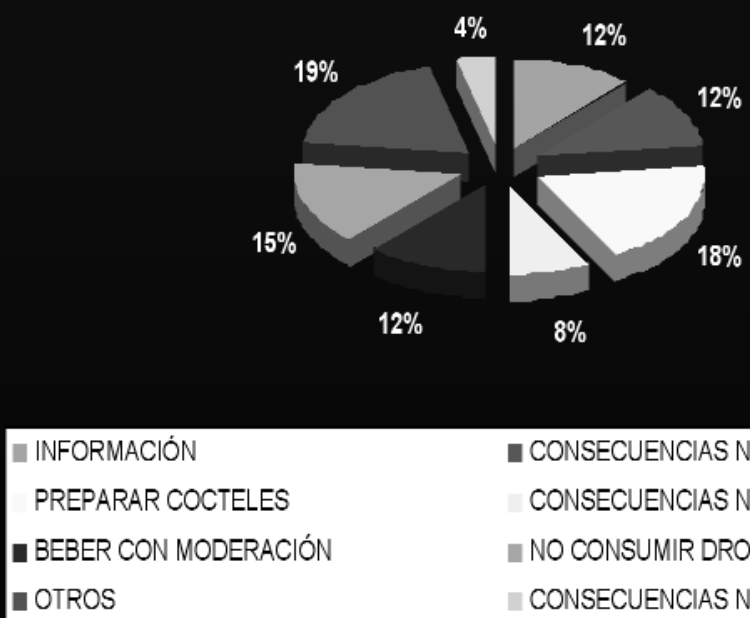

Grafico16. Aprendizajes en $3^{\circ}$ ESO

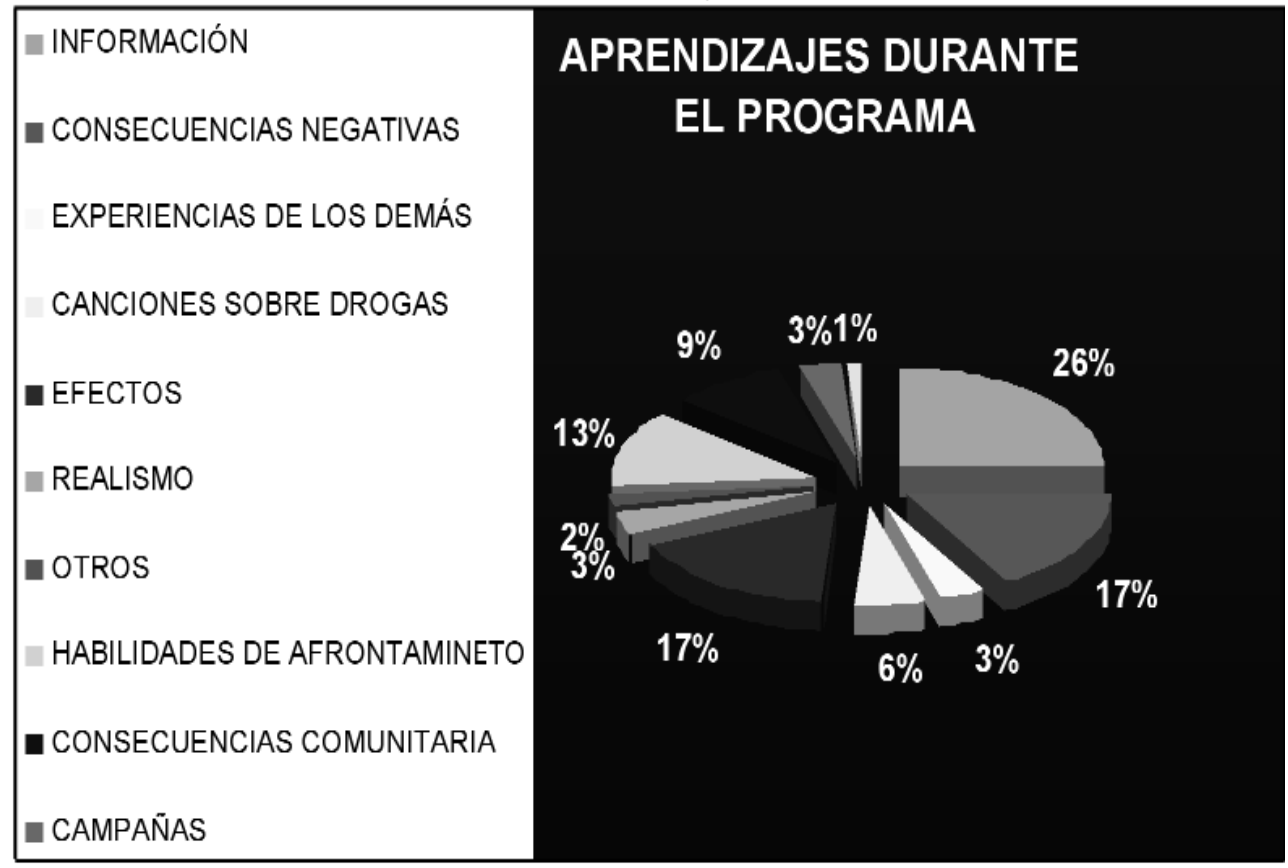


Gráfico17. Aprendizajes en $1^{\circ}$ Bachillerato

\section{APRENDIZAJES DURANTE EL PROGRAMA}

- INFORMACIÓN

- CONSECUENCIAS NEGATIVAS

HABILIDADES DE

AFRONTAMIENTO

MUSICA-DROGAS

- OTROS

$18 \%$

TRABAJAR EN GRUPO

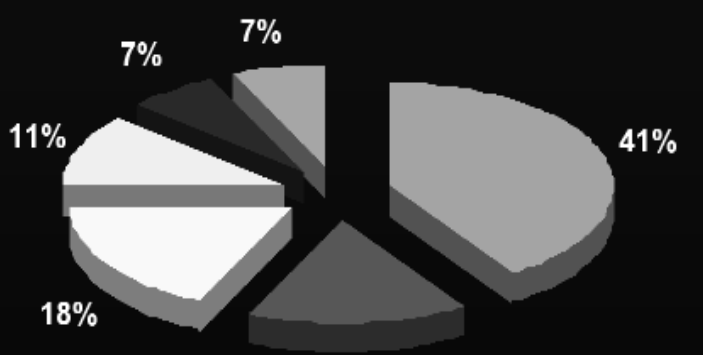

$16 \%$

Grafico 18. Propuestas de cambios en $3^{\circ}$

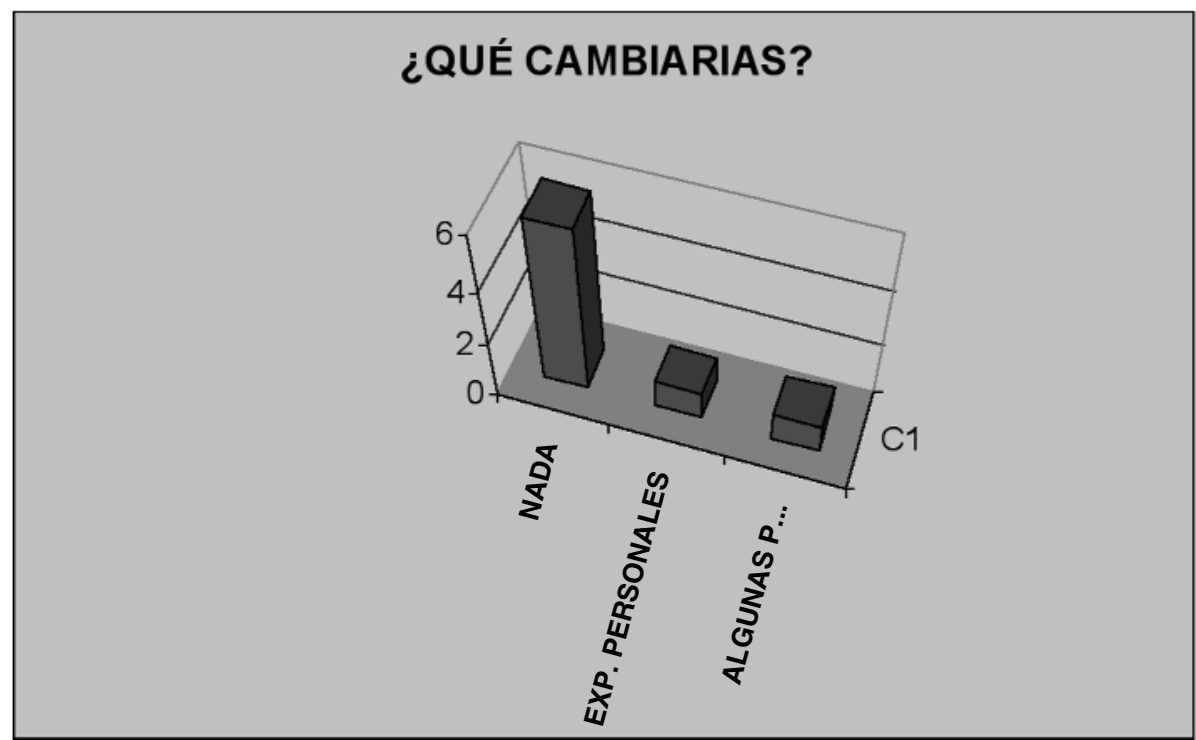


Grafico 19. Propuestas de cambios en $4^{\circ}$

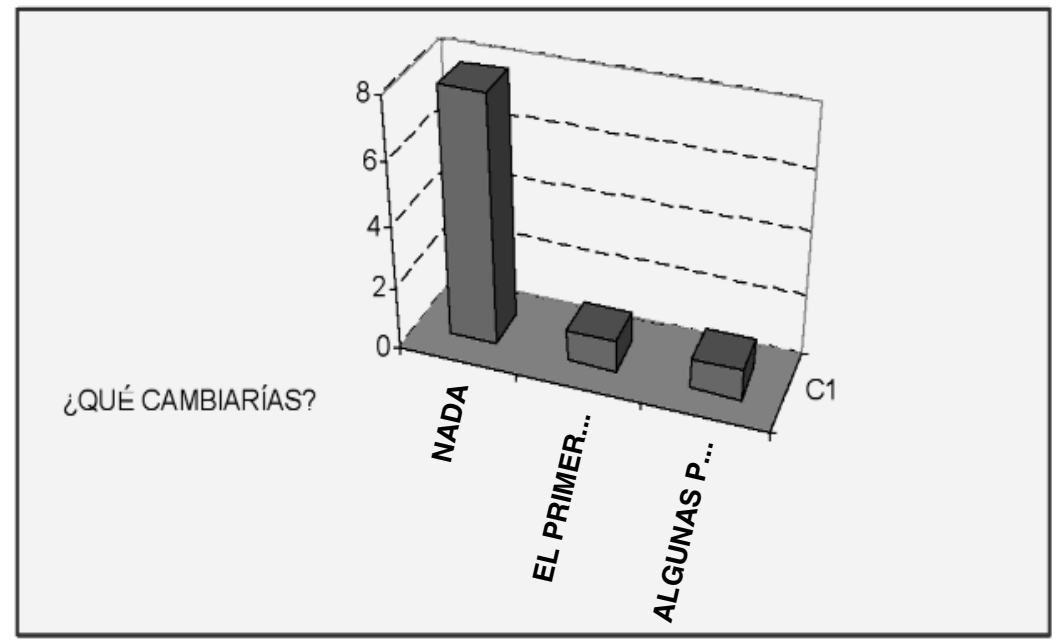

Grafico 20. Propuestas de cambios en $1^{\circ}$ Bachillerato

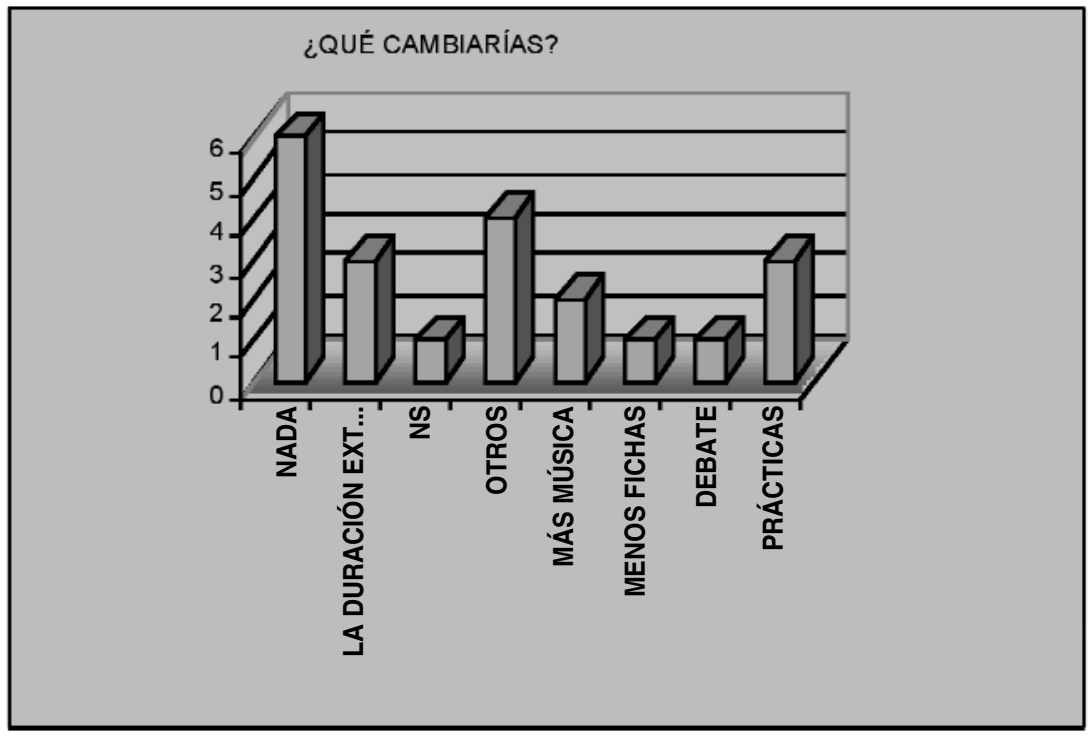

En la tabla siguiente se presentan los datos del análisis estadístico de los cuatro grupos experimentales con los que hemos trabajado:

En este caso, la pregunta se refería a si en algún momento se había planteado dejar de fumar. De los sujetos fumadores que en la situación previa al programa no se habían planteado dejar de fumar. un $15 \%$ dice que sí se lo ha planteado, aunque no lo haya intentado, y un 55 
Grafico 21. Propuestas de cambios en $2^{\circ}$ Bachillerato

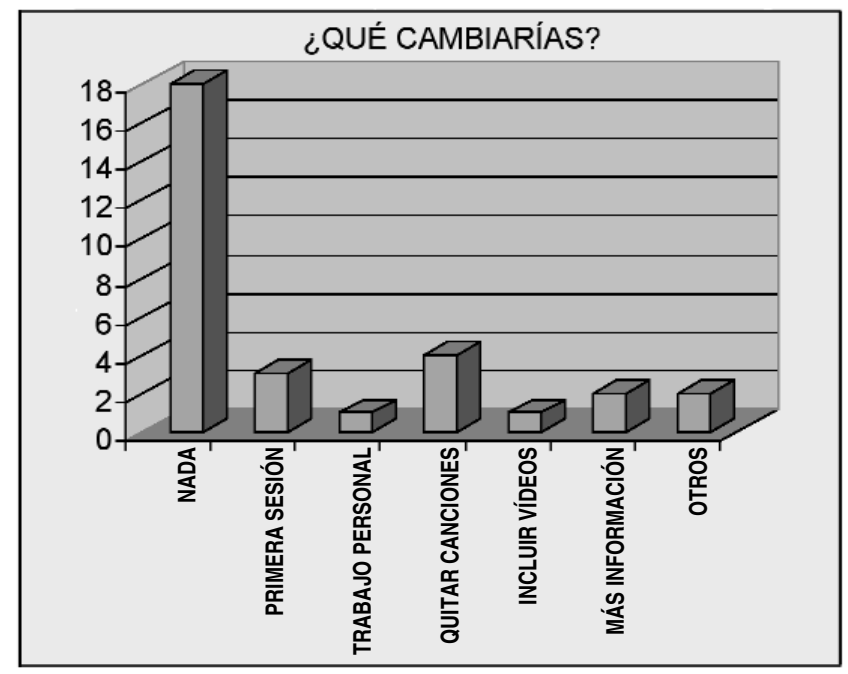

dice haberlo intentado. Es decir, del 65\% que nunca se había planteado dejar de fumar, llegamos, tras la ejecución del programa a un porcentaje del $55 \%$, consiguiéndose un aumento del deseo de dejar de fumar.

En cuanto al consumo de cannabis, en esta tabla se presentan los análisis realizados y los datos obtenidos. Es interesante comprobar que tras la aplicación del programa, el consumo de esta sustancia ha descendido. De los alumnos que antes del programa habían consumido en los 30 días anteriores, en la condición post, aumentan los porcentajes de aquellos que no consumen (42\% para los que habían consumido ? días, $50 \%$ para los que lo habían hecho 3-5 días y 40\% para los de 6-9 días).

En las siguientes tablas se presentan

Tabla 3. Datos significativos de intención de dejar de fumar

\begin{tabular}{|c|c|c|c|c|c|c|c|}
\hline & & & & $M 2: i T \Theta I$ & planteado dejar & & \\
\hline & & &, 00 & $\begin{array}{l}\mathrm{SI}, \text { lo he } \\
\text { intentado }\end{array}$ & $\begin{array}{l}\text { Si, pero nolo } \\
\text { heintentado }\end{array}$ & No & Tofai \\
\hline $\begin{array}{l}\text { ¿Te has planteado } \\
\text { dejar de fumar? }\end{array}$ &, 00 & $\begin{array}{l}\text { \% de M1: ¿Te } \\
\text { has planteado } \\
\text { dejar de fumar? }\end{array}$ & $65,0 \%$ & $.0 \%$ & , 0\% & $35,0 \%$ & $100,0 \%$ \\
\hline & Si, lo he intentado & $\begin{array}{l}\text { Recuento } \\
\% \text { de M1: ¿Te } \\
\text { has planteado } \\
\text { dejar de fumar? }\end{array}$ & $7,7 \%$ & $61,5 \%$ & $7.7 \%$ & $23,1 \%$ & $100,0 \%$ \\
\hline & No & $\begin{array}{l}\text { Recuento } \\
\text { \% de M1: ¿Te } \\
\text { has planteado } \\
\text { dejar de furnar? }\end{array}$ & $25,0 \%$ & $5,0 \%$ & $15,0 \%$ & $55,0 \%$ & $\begin{array}{r}20 \\
100,0 \%\end{array}$ \\
\hline Total & & $\begin{array}{l}\text { Recuento } \\
\% \text { de M1: ¿Te } \\
\text { has planteado } \\
\text { dejar de fumar? }\end{array}$ & $35,8 \%$ & $17,0 \%$ & $7,5 \%$ & $39,6 \%$ & 53 \\
\hline
\end{tabular}


Tabla 4. Prueba chi-cuadrado de intención de dejar de fumar

\begin{tabular}{|c|c|c|c|}
\hline \multicolumn{4}{|c|}{ Pruebas de chi-cuadrado } \\
\hline & Valor & at & $\begin{array}{c}\text { Sig. asintótica } \\
\text { (bilateral) }\end{array}$ \\
\hline Chi-cuadrado de Pearson & $33,740^{a}$ & 6 & .000 \\
\hline $\begin{array}{l}\text { Razón de verosimilitud } \\
\text { Asociación lineal por }\end{array}$ & 33,339 & 6 & .000 \\
\hline lineal & 5,007 & 1 &, 025 \\
\hline
\end{tabular}

Tabla 5. Datos significativos de frecuencia del consumo de cannabis

\begin{tabular}{|c|c|c|c|c|c|c|c|c|c|}
\hline \multirow[b]{3}{*}{$\begin{array}{l}\text { M1: frecuencia } \\
\text { de consumo } \\
\text { de cannabis }\end{array}$} & \multirow{2}{*}{.00} & \multirow{2}{*}{ Recuento } & \multicolumn{7}{|c|}{ M2: Frecuencia de consumo de cannabis } \\
\hline & & & $\frac{\text { Nunca }}{1}$ & $\frac{1 \text { a } 2 \text { dias }}{0}$ & $\frac{3 \text { a } 5 \text { dias }}{1}$ & $\frac{\text { 6a9dias }}{0}$ & $\frac{10 \text { a } 19 \text { dias }}{0}$ & $\begin{array}{l}\begin{array}{l}20 \text { o más } \\
\text { dias }\end{array} \\
0\end{array}$ & $\frac{\text { Intai }}{2}$ \\
\hline & & $\begin{array}{l}\text { \% de M1: frecuencia de } \\
\text { consumo de cannabis }\end{array}$ & $50,0 \%$ & $.0 \%$ & $50,0 \%$ & $.0 \%$ & $.0 \%$ & $.0 \%$ & $100,0 \%$ \\
\hline & Nunca & Recuento & 30 & 3 & 1 & 0 & 1 & 0 & 35 \\
\hline & & $\begin{array}{l}\text { \% de M1: frecuencia de } \\
\text { consumo de cannabis }\end{array}$ & $85,7 \%$ & $8,6 \%$ & $2,9 \%$ & $.0 \%$ & $2,9 \%$ & , 0\% & $100,0 \%$ \\
\hline & 1 a 2 dias & Recuento & 3 & 4 & 0 & 0 & 0 & 0 & 7 \\
\hline & & $\begin{array}{l}\text { \% de M1: frecuencia de } \\
\text { consumo de cannabis }\end{array}$ & $42,9 \%$ & $57,1 \%$ & $.0 \%$ & $.0 \%$ & ,0\% & $.0 \%$ & $100,0 \%$ \\
\hline & 3 a 5 dias & Recuento & 1 & 1 & 0 & 0 & 0 & 0 & 2 \\
\hline & & $\begin{array}{l}\text { \% de M1: frecuencia de } \\
\text { consumo de cannabis }\end{array}$ & $50,0 \%$ & $50,0 \%$ & $.0 \%$ & $.0 \%$ & $.0 \%$ & $.0 \%$ & $100,0 \%$ \\
\hline & 6 a 9 dias & $\begin{array}{l}\text { Recuento } \\
\% \text { de M1: frecuencia de } \\
\text { consumo de cannabis }\end{array}$ & $\begin{array}{r}2 \\
40,0 \% \\
\end{array}$ & $\begin{array}{r}1 \\
20,0 \% \\
\end{array}$ & $\begin{array}{r}0 \\
.0 \% \\
\end{array}$ & $\begin{array}{r}2 \\
40,0 \%\end{array}$ & $\begin{array}{r}0 \\
0 \%\end{array}$ & $\begin{array}{r}0 \\
.0 \%\end{array}$ & $\begin{array}{r}5 \\
100,0 \%\end{array}$ \\
\hline & 10 a 19 dias & Recuento & 0 & 0 & 0 & 0 & 1 & 1 & $\overline{2}$ \\
\hline & & $\begin{array}{l}\text { \% de M1: frecuencia de } \\
\text { consumo de cannabis }\end{array}$ & $.0 \%$ &, $0 \%$ & $.0 \%$ & $.0 \%$ & $50,0 \%$ & $50,0 \%$ & $100,0 \%$ \\
\hline & 20 o mas dias & Recuento & 0 & 0 & 0 & 0 & 1 & 1 & 2 \\
\hline & & $\begin{array}{l}\% \text { de M1: frecuencia de } \\
\text { consumo de cannabis }\end{array}$ & ,0\% & $.0 \%$ & , $0 \%$ & $.0 \%$ & $50,0 \%$ & $50,0 \%$ & $100,0 \%$ \\
\hline Total & & Recuento & 37 & 9 & 2 & 2 & 3 & 2 & 55 \\
\hline & & $\begin{array}{l}\text { \% de M1: frecuencia de } \\
\text { consumo de cannabis }\end{array}$ & $67,3 \%$ & $16,4 \%$ & $3,6 \%$ & $3,6 \%$ & $5,5 \%$ & $3,6 \%$ & $100,0 \%$ \\
\hline
\end{tabular}

Tabla 6. Prueba chi-cuadrado de frecuencia de consumo de cannabis

\begin{tabular}{|c|c|c|c|}
\hline \multicolumn{4}{|c|}{ Pruebas de chi-euadrado } \\
\hline & Valor & $\mathrm{gl}$ & $\begin{array}{l}\text { Sig. asintótica } \\
\text { (bilateral) }\end{array}$ \\
\hline Chi-cuadrado de Pearson & $90,521^{a}$ & 30 &, 000 \\
\hline Razón de verosimilitud & 49,728 & 30 &, 013 \\
\hline $\begin{array}{l}\text { Asociación lineal por } \\
\text { lineal }\end{array}$ & 26,275 & 1 &, 000 \\
\hline $\mathrm{N}$ de casos válidos & 55 & & \\
\hline
\end{tabular}

a. 8 casillas $(95,2 \%)$ tienen una frecuencia esperada inferior a 5 .

las cantidades de cannabis consumidas antes y después del programa. Como puede apreciarse, desciende significativamente las medias de consumo de esta sustancia:

En cuanto al consumo de alcohol, en las siguientes tablas encontramos los datos que corroboran que ha descendido tanto el número de borracheras, como los sujetos que llegaban a esta situación de intoxicación aguda; de igual manera se aprecia un descenso de la cantidad de alcohol consumido. 
Tabla 7. Datos significativos de cantidad de cannabis consumido Prueba para una muestra

\begin{tabular}{|c|c|c|c|c|c|c|}
\hline & \multicolumn{6}{|c|}{ Valor de orueba $=0$} \\
\hline & \multirow[b]{2}{*}{$t$} & \multirow[b]{2}{*}{ al } & \multirow[b]{2}{*}{ Sia (bilaterall } & \multirow{2}{*}{$\begin{array}{l}\text { Diferencia } \\
\text { de medias }\end{array}$} & \multicolumn{2}{|c|}{$\begin{array}{c}95 \% \text { Intervalo de } \\
\text { confianza para la } \\
\text { diferencia }\end{array}$} \\
\hline & & & & & Inferior & Sunerior \\
\hline $\begin{array}{l}\text { M1.cantidad de } \\
\text { cannabis consumida }\end{array}$ & 4,759 & 60 & .000 & 1,2787 & .7412 & 1,8162 \\
\hline $\begin{array}{l}\text { M2:cantidad de } \\
\text { cannabis consumida }\end{array}$ & 4,347 & 59 &, 000 & 8833 & ,4767 & 1,2900 \\
\hline
\end{tabular}

Tabla 8. Prueba chi-cuadrado para cantidad de cannabis consumido Estadísticos para una muestra

\begin{tabular}{|l|r|r|r|r|}
\hline & $\mathrm{N}$ & Media & \multicolumn{1}{c|}{$\begin{array}{c}\text { Desviación } \\
\text { tío. }\end{array}$} & $\begin{array}{c}\text { Error típ. de } \\
\text { lamedia }\end{array}$ \\
\hline $\begin{array}{l}\text { M1.cantidad de } \\
\text { cannabis consumida }\end{array}$ & 61 & 1,2787 & 2,09866 &, 26871 \\
\hline $\begin{array}{l}\text { M2:cantidad de } \\
\text { cannabis consumida }\end{array}$ & 60 &, 8833 & 1,57407 &, 20321 \\
\hline
\end{tabular}

Tabla 9. Datos significativos de cantidad de alcohol consumido Estadísticos para una muestra

\begin{tabular}{|l|r|c|c|c|}
\hline & $\mathrm{N}$ & Media & $\begin{array}{c}\text { Desviación } \\
\text { tía }\end{array}$ & $\begin{array}{c}\text { Error típ. de } \\
\text { la media }\end{array}$ \\
\hline $\begin{array}{l}\text { M1:cantidad de } \\
\text { alcohol consumida }\end{array}$ & 61 & 8,1803 & 11,05367 & 1,41528 \\
\hline $\begin{array}{l}\text { M2: cantidad de } \\
\text { alcohol consumida }\end{array}$ & 57 & 4,1404 & 4,99442 &, 66153 \\
\hline
\end{tabular}

Tabla 10. Prueba chi-cuadrado para cantidad de alcohol consumido

Prueba para una muestra

\begin{tabular}{|c|c|c|c|c|c|c|}
\hline & \multicolumn{6}{|c|}{ Valor de orueba $=0$} \\
\hline & \multirow[b]{2}{*}{$t$} & \multirow[b]{2}{*}{ al } & \multirow[b]{2}{*}{ Sio (bilateral) } & \multirow{2}{*}{$\begin{array}{l}\text { Diferencia } \\
\text { de medias }\end{array}$} & \multicolumn{2}{|c|}{$\begin{array}{c}95 \% \text { Intervalo de } \\
\text { confianza para la } \\
\text { diferencia }\end{array}$} \\
\hline & & & & & Inferior & Sunerior \\
\hline $\begin{array}{l}\text { M1: cantidad de } \\
\text { alcohol consumida }\end{array}$ & 5,780 & 60 & .000 & 8,183 & 5,3494 & 11,0113 \\
\hline $\begin{array}{l}\text { M2: cantidad de } \\
\text { alcohol consumida }\end{array}$ & 6,259 & 56 &, 000 & 4,1404 & 2,8152 & 5,4655 \\
\hline
\end{tabular}


Tabla 11. Datos significativos de borracheras en los últimos 30 dias

Tabla de contingencia M1: ¿Te has emborrachado en los últimos 30 días? * M2: ¿Te has emborrachado en los últimos 30 días?

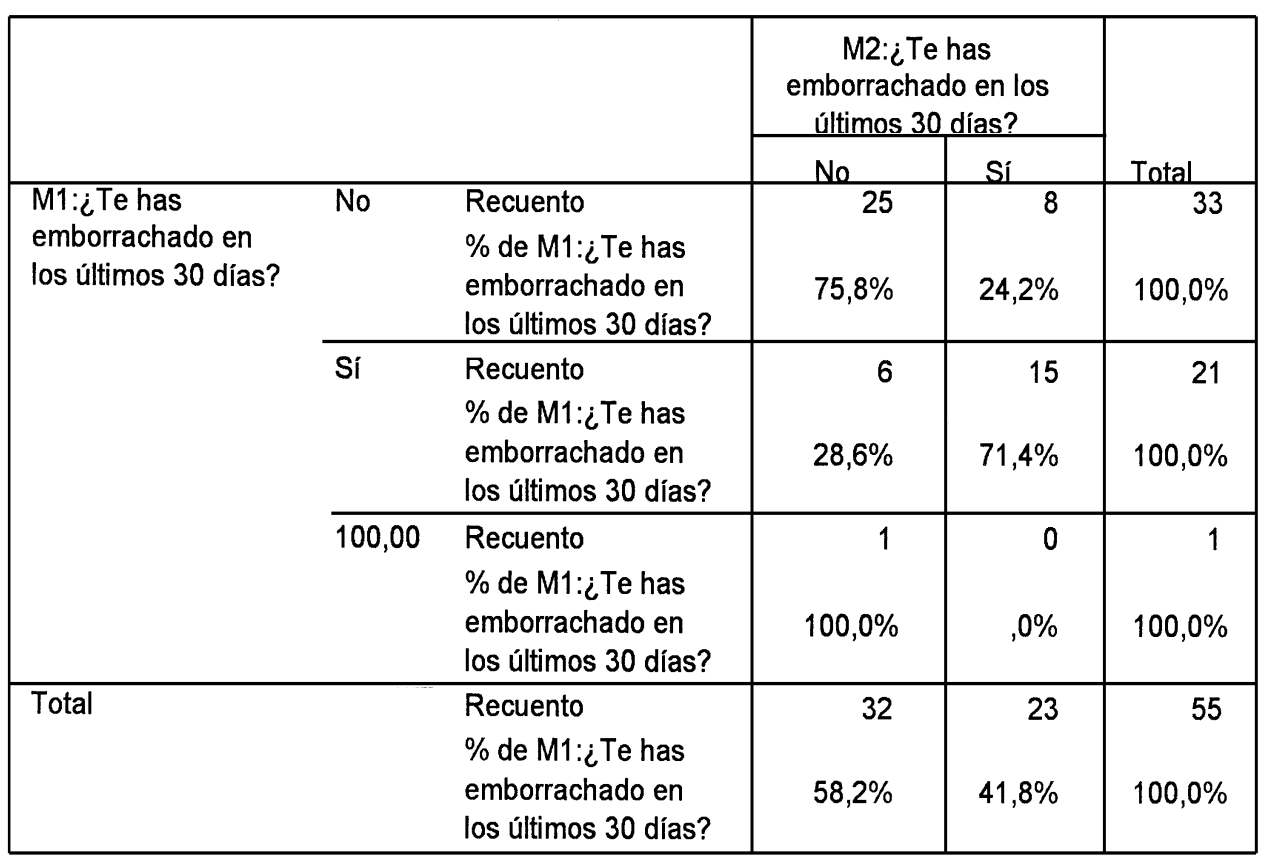

Tabla 12. Prueba chi-cuadrado de borracheras en los últimos 30 días

Pruebas de chi-cuadrado

\begin{tabular}{|c|c|c|c|}
\hline & Valor & $\mathrm{gl}$ & $\begin{array}{c}\text { Sig. asintótica } \\
\text { (bilateral) }\end{array}$ \\
\hline Chi-cuadrado de Pearson & $25,076^{a}$ & 6 &, 000 \\
\hline Razón de verosimilitud & 18,402 & 6 &, 013 \\
\hline $\begin{array}{l}\text { Asociación lineal por } \\
\text { lineal }\end{array}$ & 13,293 & 1 &, 000 \\
\hline $\mathrm{N}$ de casos válidos & 55 & & \\
\hline
\end{tabular}

a. 8 casillas $(66,7 \%)$ tienen una frecuencia esperada inferior a 5 . La frecuencia mínima esperada es 0,9 .

Un $30 \%$ de los sujetos que antes de "Prevenir en otra onda" se habían emborrachado en los últimos 30 días, ha dejado de hacerlo.

Por último, presentamos los datos obtenidos en cuanto a la percepción del grado de información que se tiene de la temática de las drogas. El cambio más significativo tras la aplicación del programa está en aquellos sujetos que antes se consideraban poco informados 
Tabla 13. Datos significativos de percepción de la información que se posee sobre drogas

\begin{tabular}{|c|c|c|c|c|c|c|}
\hline & & & \multicolumn{3}{|c|}{$\begin{array}{l}\text { M2: ¿Te sientes sufficientemente } \\
\text { informado sobre drogas? }\end{array}$} & \multirow[b]{2}{*}{ Total } \\
\hline & & & $\begin{array}{c}\mathrm{Si}_{1} \\
\text { perfectamente }\end{array}$ & $\begin{array}{c}\mathrm{Si}, 10 \\
\text { suficiente }\end{array}$ & $\begin{array}{l}\text { Solo a } \\
\text { medias }\end{array}$ & \\
\hline \multirow[t]{4}{*}{$\begin{array}{l}\text { M1: ¿Te sientes } \\
\text { suficientemente } \\
\text { informado sobre } \\
\text { drogas? }\end{array}$} & Si, perfectamente & $\begin{array}{l}\text { Recuento } \\
\text { \% de M1: ¿Te sientes } \\
\text { suficientemente } \\
\text { informado score drogas? }\end{array}$ & $56,3 \%$ & $43,8 \%$ & $.0 \%$ & $\begin{array}{r}16 \\
100,0 \%\end{array}$ \\
\hline & Si, lo suficiente & $\begin{array}{l}\text { Recuento } \\
\text { \% de M1: ¿Te sientes } \\
\text { suficientemente } \\
\text { informado sobre drogas? }\end{array}$ & $\begin{array}{r}8 \\
26,7 \%\end{array}$ & $\begin{array}{r}21 \\
70,0 \%\end{array}$ & $\begin{array}{r}1 \\
3,3 \%\end{array}$ & $\begin{array}{r}30 \\
100,0 \%\end{array}$ \\
\hline & Solo a medias & $\begin{array}{l}\text { Recuento } \\
\text { \% de M1: ¿Te sientes } \\
\text { suficientemente } \\
\text { informado sobre drogas? }\end{array}$ & $\begin{array}{r}1 \\
12,5 \%\end{array}$ & $\begin{array}{r}4 \\
50,0 \%\end{array}$ & $\begin{array}{r}3 \\
37,5 \%\end{array}$ & $\begin{array}{r}8 \\
100,0 \%\end{array}$ \\
\hline & No, estoy mal informado & $\begin{array}{l}\text { Recuento } \\
\text { \% de M1: ¿Te sientes } \\
\text { suficientemente } \\
\text { informado sobre drogas? }\end{array}$ & $\begin{array}{l}0 \\
.0 \%\end{array}$ & $\begin{array}{r}0 \\
.0 \%\end{array}$ & $\begin{array}{r}1 \\
100,0 \%\end{array}$ & $\begin{array}{r}1 \\
100,0 \%\end{array}$ \\
\hline \multicolumn{2}{|l|}{ Total } & $\begin{array}{l}\text { Recuento } \\
\text { \% de M1: ¿Te sientes } \\
\text { suficientemente } \\
\text { informado sobre drogas? }\end{array}$ & $\begin{array}{r}18 \\
32,7 \%\end{array}$ & $58,2 \%$ & $9,1 \%$ & $\begin{array}{r}55 \\
100,00 \%\end{array}$ \\
\hline
\end{tabular}

y después creen que tienen suficiente información o están adecuadamente informados. $50 \%$ y $12 \%$ respectivamente). Así, aumenta sustancialmente la información que el joven dice tener sobre drogas.

Pero también se completa la información que ya tenían. Así, los jóvenes que decían estar informados suficientemente antes y después pasan a estar perfectamente informados, suponen un porcentaje del 27\%. Algo similar ocurre con la utilidad de la información. Es decir, aumentan los porcentajes de los alumnos que consideran que la información que poseen es muy útil.
Relacionado con el tema de la información, encontramos que el $80 \%$ de los alumnos que en el pretest contestan que los beneficios de la droga son superiores a los riesgos, contestan negativamente a la misma pregunta después de vivir la situación experimental.

\section{CONCLUSIONES}

Una de las primeras conclusiones que podemos establecer del análisis desarrollado es que sin lugar a dudas, el programa "Prevenir en otra onda" consigue unos resultados satisfactorios en la consecución de unos de sus objetivos y fun- 
Tabla 14. Datos significativos de la utilidad de la información que se posee sobre drogas

Tabla de contingencia M1: Utilidad de la información. M2: utilidad de la información

\begin{tabular}{|c|c|c|c|c|c|c|c|c|}
\hline & & & \multicolumn{5}{|c|}{ M2: Utilidad de la información } & \multirow[b]{2}{*}{ Total } \\
\hline & & & Muy útil & Bastante útil & Poco útil & Nada útil & $\begin{array}{c}\text { No sé qué } \\
\text { valoración } \\
\text { hacer }\end{array}$ & \\
\hline \multirow[t]{5}{*}{$\begin{array}{l}\text { M1: utilidad } \\
\text { de la } \\
\text { información }\end{array}$} & & $\begin{array}{l}\text { \% de M1: Utilidad } \\
\text { de la información }\end{array}$ & $\begin{array}{r}5 \\
71,4 \%\end{array}$ & $\begin{array}{r}2 \\
28,6 \%\end{array}$ & $\begin{array}{r}0 \\
.0 \%\end{array}$ & $\begin{array}{r}0 \\
.0 \%\end{array}$ & $\begin{array}{r}0 \\
.0 \%\end{array}$ & $\begin{array}{r}7 \\
100,0 \%\end{array}$ \\
\hline & Bastante util & $\begin{array}{l}\text { Recuento } \\
\text { \% de M1: Utilidad } \\
\text { de la información }\end{array}$ & $\begin{array}{r}6 \\
20,7 \%\end{array}$ & $\begin{array}{r}19 \\
65,5 \%\end{array}$ & $\begin{array}{r}2 \\
6,9 \% \\
\end{array}$ & $\begin{array}{r}0 \\
.0 \%\end{array}$ & $\begin{array}{r}2 \\
6,9 \%\end{array}$ & $\begin{array}{r}29 \\
100,0 \%\end{array}$ \\
\hline & Poco util & $\begin{array}{l}\text { Recuento } \\
\text { \% de M1: Utilidad } \\
\text { de la información }\end{array}$ & $\begin{array}{r}0 \\
.0 \% \\
\end{array}$ & \begin{tabular}{r|}
6 \\
$66,7 \%$ \\
\end{tabular} & \begin{tabular}{r|}
2 \\
$22,2 \%$ \\
\end{tabular} & $\begin{array}{r}1 \\
11,1 \%\end{array}$ & $\begin{array}{r}0 \\
.0 \%\end{array}$ & $\begin{array}{r}9 \\
100,0 \%\end{array}$ \\
\hline & Nada utı & $\begin{array}{l}\text { Recuento } \\
\% \text { de M1: Utilidad } \\
\text { de la información }\end{array}$ & $\begin{array}{r}0 \\
.0 \%\end{array}$ & $\begin{array}{r}2 \\
100,0 \% \\
\end{array}$ & \begin{tabular}{r|}
0 \\
$.0 \%$
\end{tabular} & \begin{tabular}{r|}
0 \\
$.0 \%$
\end{tabular} & $\begin{array}{r}0 \\
.0 \%\end{array}$ & $\begin{array}{r}2 \\
100,0 \%\end{array}$ \\
\hline & $\begin{array}{l}\text { No se que } \\
\text { valoracion hacer }\end{array}$ & $\begin{array}{l}\text { Recuento } \\
\text { \% de M1: Utilidad } \\
\text { de la información }\end{array}$ & $\begin{array}{r}2 \\
25,0 \% \\
\end{array}$ & \begin{tabular}{r|}
5 \\
$62,5 \%$ \\
\end{tabular} & \begin{tabular}{r|}
1 \\
$12,5 \%$ \\
\end{tabular} & \begin{tabular}{r|}
0 \\
$.0 \%$
\end{tabular} & $\begin{array}{r}0 \\
.0 \%\end{array}$ & $\begin{array}{r}8 \\
100,0 \%\end{array}$ \\
\hline Total & & $\begin{array}{l}\text { Recuento } \\
\% \text { de M1: Utilidad } \\
\text { de la información }\end{array}$ & $\begin{array}{r}13 \\
23,6 \%\end{array}$ & \begin{tabular}{r|}
34 \\
$61,8 \%$ \\
\end{tabular} & $\begin{array}{r}5 \\
9,1 \%\end{array}$ & $\begin{array}{r}1 \\
1,8 \%\end{array}$ & $\begin{array}{r}2 \\
3,6 \%\end{array}$ & $\begin{array}{r}55 \\
100,0 \%\end{array}$ \\
\hline
\end{tabular}

Tabla 15. Datos significativos de beneficios atribuidos al consumo de drogas

Tabla de congtingencia M1: Beneficios atriburdos al consumo de drogas. M2: Beneficios atribuídos al consumo de drogas

\begin{tabular}{|c|c|c|c|c|c|}
\hline \multirow{6}{*}{$\begin{array}{l}\text { M1: Beneficios atribuídos } \\
\text { al consumo de drogas }\end{array}$} & \multirow{4}{*}{ no } & \multirow{4}{*}{$\begin{array}{l}\text { Recuento } \\
\% \text { de } M 1 \text { : Beneficios } \\
\text { atribuídos al } \\
\text { consumo de drogas }\end{array}$} & \multicolumn{2}{|c|}{$\begin{array}{c}\text { M2: Beneficios } \\
\text { atribuidos al consumo } \\
\text { de droaas }\end{array}$} & \multirow[b]{2}{*}{ Total } \\
\hline & & & no & $\mathrm{Si}$ & \\
\hline & & & 45 & 5 & 50 \\
\hline & & & $90,0 \%$ & $10,0 \%$ & $100,0 \%$ \\
\hline & $\mathrm{Si}$ & Recuento & 4 & 1 & 5 \\
\hline & & $\begin{array}{l}\% \text { de } M 1: \text { Beneficios } \\
\text { atribuidos al } \\
\text { consumo de drogas }\end{array}$ & $80,0 \%$ & $20,0 \%$ & $100,0 \%$ \\
\hline Total & & Recuento & 49 & 6 & 55 \\
\hline & & $\begin{array}{l}\% \text { de } M 1: \text { Beneficios } \\
\text { atribuídos al } \\
\text { consumo de drogas }\end{array}$ & $89,1 \%$ & $10,9 \%$ & $100,0 \%$ \\
\hline
\end{tabular}

damentos teóricos y metodológicos de partida: aumentar la significatividad y relevancia de los mensajes preventivos y educativos a establecer con los más jóvenes por la utilización de un lenguaje que es sumamente conocido y valorado por estos jóvenes: la música que escuchan, en la que invierten tiempo y dinero, y que les transmite un gran número de sensaciones positivas, muchas veces escasamente analizadas o utilizadas pese a su potencialidad.

En este sentido, se confirma aquello 
que seña Funes (1996) al hablar del trabajo con adolescentes de la necesidad de hablar en sus lenguajes si pretendemos trabajar con jovenes. También se confirma, como estrategia preventiva y educativa, que lo lúdico es un excelente material de trabajo para desarrollar acercamientos en materia de prevención de drogas, ya que aprender jugando siempre ha sido y sigue siendo una estrategia fundamental y muy útil en casi todas las edades pero especialmente en edades como aquellas a las que dirigimos el presente proyecto preventivo.

La música además de entretenernos permite acceder al mundo propio de los jóvenes, a algunas de sus expresiones características, a espacios significativos de comunicación con ellos, se trata de ponernos en onda, en su onda, en conexión, en comunicación.

Que podamos afirmar la confirmación de este particular tiene mucho que ver con las aportaciones al cuestionario interno de carácter cualitativo en el cual los participantes del programa valoran el mismo manifestando elevadas dosis de satisfacción y significatividad con el mismo.

Otro elemento a valorar especialmente del programa es la valoración que hacen los participantes con respecto a la contribución del programa para que tomasen decisiones con respecto a las drogas en unos casos reafirmando su decisión de no consumir y en otros estableciendo un rechazo a los consumos, modificando expectativas, aportando informaciones y generando actitudes.

También cabe señalar como un elemento no medido en el cuestionario pre y post pero sí en el cuestionario interno, como es la adquisición de habilidades para la toma de decisiones en situacio- nes de consumo o la resistencia a las mismas, obtiene resultados positivos de valoración en la línea de que los participantes en el programa señalan que el mismo contribuyó a dotarles de más estrategias y habilidades para enfrentarse y gestionar situaciones de posible riesgo de consumo.

Otro elemento clave a destacar, tiene que ver con la valoración que se hace del monitor que ejecuto el programa, muchas veces clave de una correcta ejecución de los programas de prevención.

Como se señala en las bases teóricas que establecemos para el desarrollo del presente programa creemos que el mismo consigue, en función de los resultados obtenidos, realzar los factores de protección y disminuir los factores de riesgo, se adapta especialmente a los conocimientos, situaciones y percepciones de los destinatarios, incluyen la consideración de sustancias y su trato, considera la utilización de métodos interactivos y la participación de los jóvenes. Todos estos elementos del programa, son además, los más valorados por los participantes con lo que se consigue una más que interesante confluencia entre los planteamientos teóricos, las evaluaciones concretas del programa y el desarrollo práctico del mismo que garantiza la coherencia interna, teórica y empírica de la propuesta de intervención desarrollada y pilotada.

Es importante en toda evaluación y en todo programa de prevención que exista una clara conexión entre los principios teóricos que se plantean como elementos de partida y la plasmación de los mismos en acciones concretas evaluadas posteriormente. Así, los principios teóricos han servido sin duda para desarrollar el programa y muy especialmente el establecimiento de métodos interactivos que propician la participación de los jóvenes, 
su implicación mediante al utilización de iguales (como las últimas sesiones de cada una de las aplicaciones), métodos que propician también la oportunidad para practicar las nuevas habilidades adquiridas a través de su ejecución práctica (como la cuarta sesión del programa) 0 intervenciones que permitan llegar a jóvenes diversos, diferentes en sus estilos de vida, gustos, preferencias y pautas de consumo.

En otro orden de cosas, los datos del análisis estadístico preliminar que hemos realizado, confirman que "Prevenir en otra onda" no solo es un programa que engancha con los jóvenes sino que además en su ejecución como programa piloto consiguió buenos resultados en cuanto a algunas de las variables cuantitativas que habiamos contemplado. Los jóvenes que vivieron nuestro programa disminuyeron sus consumos de alcohol y cannabis y las veces que los consumos de alcohol conducen a la borrachera.

Pero nos son los únicos cambios que se producen. También aumentan considerablemente el número de alumnos que se plantean dejar de fumar, los que se consideran bastante bien informados sobre el tema de drogas y aquellos que creen que está información es de gran utilidad. Y, sobre todo, los que perciben que en el tema de las drogas son más los riesgos que los beneficios.

Pero creemos que no son los únicos cambios que vamos a encontrar. Por ello, en el análisis estadístico detallado que se realizará a continuación contemplaremos también otras variables que pueden ser significativas, como la percepción de riesgo o la tendencia al no consumo. En este mismo estudio, no solo se presentarán los datos generales, sino análisis de los datos de cada uno de los grupos. Este es uno de los elementos a completar.

Entre los elementos a mejorar, encontramos la necesidad de reestructurar alguna de las sesiones, como la primera sesión de primero de bachillerato, una de las que peor valoración tuvo con el agravante de que el grupo escogido como modelo desarrollo una campaña de promoción publicitaria de una bebida alcohólica una vez que ya teníamos diseñado el programa y en plena ejecución del mismo, también resulta necesario desarrollar e implementar actuaciones que consideren a los padres y madres de los jóvenes participantes.

Otro elemento especialmente a considerar y que nos generó algunos problemas técnicos y de ejecución, tienen que ver con el manejo en un programa universal como éste de demandas de información sobre drogas de jóvenes que están en consumo activo actual de sustancias frente a sus compañeros que no se encuentran en esta situación de consumo; otra de las dificultades tiene que ver con la escasez de canciones con mensajes preventivos a utilizar en el desarrollo de las actividades preventivas. 
Prevenir en otra onda

\section{BIBLIOGRAFÍA}

Asun, D. y Alvarado, R. (1991). "Aspectos médicos y sociales del consumo de drogas". En: D. Asun, J. Alfaro, R. Alvarado y G. Morales (Eds.), Drogas, juventud y exclusión social. Santiago de Chile: Universidad Diego Portales.

Calafat, A. Bohrn, K., Juan M, Kokkevi A, Maalsté, N., (1999). "Night life in Europe and recreative drug use" Sonar 98, Palma de Mallorca: IREFREA ESPAÑA.

Calafat, A., Amengual, M. (1999). "Actuar es posible: Educación sobre el alcohol”. Madrid: Plan Nacional sobre Drogas. Secretaría General Técnica.

Calafat, A., Fernández, C., Juan, M., Bellis, A., Bohrn, K. Hakkarainen, P., Kilfoyle- Carrington, M., Kokkevi, A., Maalsté N., Mendes, F. Siamou I., Simon J., Stocco P. Y Zavatti, P. (2001). "Risk and control in the recreational drug culture" Sonar, 2000, Palma de Mallorca: IREFREA. ESPAÑA.

CAM, (1991). "La Juventud en la comunidad de Madrid: Conocer para actuar". Madrid: CAM.

Cami Morell J. Extasis y la ruta del bakalao/ entidad/es del autor/es: universidad autonoma de barcelona.catedra de farmacologia

Company, S. Y Herranz, C. (1994). "Música y drogas" Curso de experto en drogodependencias FAD.

Congreso "Jóvenes, Noche y Alcohol" (2002). Delegación del Gobierno para el Plan Nacional Sobre Drogas. Madrid, Palacio de Congresos. 1214 de Febrero de 2002.

De los Ríos, A. Y Sánchez, M. (1997). "Habla, grita, canta, que piensas de ti, de tu familia, de tu ciudad, de las drogas" editorial Surgir, Colombia.

Del Campo, S. (1997). "La juventud europea, desvalida y desentendida" Cuenta y razón del pensamiento actual. Construir Europa juntos.

Elzo, J. (1996). "Los valores en la Comunidad Autónoma del País Vasco y Navarra, SU evolución en los años 1990-1995", Vitoria Gasteiz, Gobierno Vasco.

Elzo, J. (1999). "Tiempo libre : alcohol cannabis y musica" Revista Proyecto Hombre, 1999 .- n. 32; p. $22-25$.
Elzo, J. (2002). "¿Qué está pasando en la noche?" del Congreso sobre "Jóvenes, Noche y Alcohol" organizado por la Delegación del Gobierno para el Plan Nacional Sobre Drogas. Madrid, Palacio de Congresos. 12- 14 de Febrero de 2002.

Elzo, J. Orizo, F. A. y otros (1999). "Jóvenes españoles 1999”. Madrid: ed. Fundación Santa Maria. SM ediciones.

Encuesta a la población escolar, 2000. PND.

Feixa, C. (1998). "De jóvenes, bandas y tribus. Antropología de la juventud" Barcelona, Ariel.

Fouce, J. G.(2003). "Música y drogodependencias: análisis de algunos tópicos encontrados en la música".Revista Adicciones, vol 15. Num 3.

Fuertes, J.C. , Palacios, F. A. (1999). "Hablemos sobre las drogas”. Madrid: ed. Arán. C.A.M. Agencia Antidroga y Asociación Carpe Diem.

Funes, J. (1996). "Drogas y adolescentes" Madrid: Editorial Aguilar.

Gamella, J.F., Álvarez Roldan, A., Romo, N. (1997). "La "fiesta" y el "éxtasis" drogas de sintesis y nuevas culturas juveniles". En Estudios de Juventud, № 40, 17-22.

Gilman de Lifeline M. (1994). "Nuevo fenómeno de la música y la droga" en opin juv, 1994 .- n. 4; $p$. $4-6$.

Gómez Pérez, R. (1994). "El rock. Historia y análisis del movimiento cultural más importante del siglo XX.”. Madrid, El Drac.

Injuve septiembre (2001). "La noche: un conflicto de poder"

Laporta, F. J. (2000). "El cansancio de la democracia" en Claves de la razón práctica, nº99, p. 20-25.

Laporta, F. J. (2000). "La incubación" El País, Jueves 16 marzo, 2000.

Levices Mallo J. (1986). "Modas musicales condiciones sociales" Madrid: direccion general de juventud.

Levices Mallo J.J.,Serrano Pascual A. (1993). "Estructura social y estructura musical" Rev. Int. Soc., 1993 .- n. 6; p. 5-26. 
Llopis Llacer j.j. (1997). "Drogas de diseño : nuevos usos en una nueva era" Rev. socidrogalco hol en : entre todos, n. 14; p. 120-122 6 refs.

Martin Serrano, M. Y Velarde Hermida, O. (2001). “Juventud en España 2000”. Madrid: INJUVE.

Mayor. J. (1996). "Modelo sintético para comprender el proceso de drogadicción”. Proyecto hombre $n^{-}$19. Madrid.

Megias Quiros I.,Rodriguez San julian (2000). "Musica y jovenes : identidades, diferenciaciones y referentes de ocio" Revista Estudios de Juventud, 2000 .- n. 50; p. 53-61.

Megias Quirós, I., Rodríguez E. (2003). ”Jóvenes entre sonidos" F.A.D e I.N.J.U.V.E.

Navarro, M. Y Mateo, M. J. (1994). "Jóvenes y juventud" Documentación social, nº 95. Abril- junio 1994, p, 9-23.

Pons, J., Berjano, E. (1999). "El consumo abusivo de alcohol en la Adolescencia: un modelo explica tivo desde la psicología social”. Madrid: Ministerio del Interior. Plan Nacional sobre Drogas. Edex.

Programa Órdago, el desafió de vivir sin drogas,

Recio, P. D. (1997). "El fenómeno de la droga como capital simbólico y hecho social total”. En Estudios de Juventud. № 40. Pág. 9-16.

Ríos A.M. De Los, Sanchez M.M. (1997). "Habla grita canta: que piensas de ti de tu familia tu ciudad de las drogas".

Salustiano del Campo, (1997). "La juventud europea, desvalida y desentendida” Cuenta y razón del pensamiento actual. Construir Europa juntos.

Sánchez, J. y González, T. (1995). "El ritmo de la noche” Revista Trafico. Abril 1995. Pág. 8-15).

Sedo, C. (1999). "Jovenes drogas y rock'n"” Revista proyecto hombre, 1999 .- n. 32; p. 40-42.

Walter, B. Y Gruber M. (1997). "Europa : la droga y los violines" Revista Opnion Juvenil 1997 .n. $14 ;$ p. 12-13. 